\title{
Invariants of Four-Manifolds with Flows Via Cohomological Field Theory
}

\author{
Dedicated to John Milnor on his $80^{\text {th }}$ birthday
}

\author{
Hugo García-Compeán ${ }^{a 1}$, Roberto Santos-Silva ${ }^{a 2}$, Alberto Verjovsky ${ }^{b 3}$ \\ ${ }^{a}$ Departamento de Física, Centro de Investigación y de Estudios Avanzados del IPN \\ P.O. Box 14-740, 07000 México D.F., México \\ ${ }^{b}$ Instituto de Matemáticas, UNAM, Unidad Cuernavaca \\ Av. Universidad $s / n$, Col. Lomas de Chamilpa \\ c.p. 62210, Cuernavaca Morelos, México
}

\begin{abstract}
The Jones-Witten invariants can be generalized for non-singular smooth vector fields with invariant probability measure on 3-manifolds, giving rise to new invariants of dynamical systems [22]. After a short survey of cohomological field theory for Yang-Mills fields, Donaldson-Witten invariants are generalized to four-dimensional manifolds with non-singular smooth flows generated by homologically non-trivial $p$-vector fields. These invariants have the information of the flows and they are interpreted as the intersection number of these flow orbits and constitute invariants of smooth four-manifolds admitting global flows. We study the case of Kähler manifolds by using the Witten's consideration of the strong coupling dynamics of $\mathcal{N}=1$ supersymmetric Yang-Mills theories. The whole construction is performed by implementing the notion of higher dimensional asymptotic cycles à la Schwartzman [18]. In the process Seiberg-Witten invariants are also described within this context. Finally, we give an interpretation of our asymptotic observables of 4-manifolds in the context of string theory with flows.
\end{abstract}

\footnotetext{
${ }^{1}$ e-mail address: compean@fis.cinvestav $\cdot m x$

${ }^{2}$ e-mail address: rsantos@fis.cinvestav.mx

${ }^{3}$ e-mail address: alberto@matcuer.unam.mx
} 


\section{Introduction}

Quantum field theory is not only a framework to describe the physics of elementary particles and condensed matter systems but it has been useful to describe mathematical structures and their subtle interrelations. One of the most famous examples is perhaps the description of knot and link invariants through the correlation functions of products of Wilson line operators in the Chern-Simons gauge theory [1]. These invariants are the Jones-Witten invariants or Vassiliev invariants depending on whether the coupling constant is weak or strong respectively. Very recently some aspects of gauge and string theories found a strong relation with Khovanov homology [2].

In four dimensions the Donaldson invariants are invariants of the smooth structure on a closed four-manifold. This is in the sense that if two homeomorphic differentiable manifolds have different Donaldson invariants then they are not diffeomorphic $[3,4]$. These invariants were reinterpreted by Witten in terms of the correlation functions of suitable observables of a cohomological Yang-Mills field theory in four dimensions [5]. Such a theory can be obtained from an appropriate topological twist on the global symmetries of the $\mathcal{N}=2$ supersymmetric Yang-Mills theory in Minkowski space with global $R$-symmetry $\mathrm{SU}(2)$ that rotates the supercharges. A gravitational analog of the Donaldson theory is given by the topological gravity in four and two dimensions [6]. The computation of Donaldson invariants for Kähler manifolds has been done from the mathematical point of view in Refs. [7, 8]. These Donaldson invariants were later reproduced in Ref. [9] by using the strong coupling dynamics of $\mathcal{N}=1$ supersymmetric gauge theories in four dimensions. Precisely a deeper understanding of the dynamics of strong coupling $\mathcal{N}=2$ supersymmetric Yang-Mills theories in four dimensions [10] including the notion of $S$-duality, allowed to give an alternative approach to Donaldson theory in terms of the low energy effective abelian gauge theory coupled to magnetic monopoles [11]. For a recent account of all these developments, see [12].

Moreover, the topological twist was applied to other theories such as string theory resulting in the so called topological sigma models [13]. The two possible twists of the global symmetries of the world-sheet theory leads to the so called $A$ and $B$-models, whose correlation functions give rise to a description of the moduli space in terms of only the Kähler cone or only the moduli of complex structures of a target space CalabiYau manifold. A-models give rise to Gromov-Witten invariants. Mirror symmetry is realized through the interchanging of $A$ and $B$ models of two Calabi-Yau manifolds related by the interchanging of Betti numbers [14]. For a recent survey of all these topological field theories and their interrelations, see for instance [15].

On the other hand it is well known that topology and symplectic geometry play a very important role in the theory of dynamical systems [16]. Schwartzman introduced some years ago, homology 1-cycles associated to a foliation known as asymptotic cycles [17]. These 1-cycles are genuine homology cycles and they represent an important tool to study some properties of dynamical systems. Moreover, the generalization to $p$-cycles, with $p>1$, was done in Ref. [18]. Such generalization was achieved by using some concepts of dynamical systems such as flow boxes and geometric currents $[19,20]$. The definition of asymptotic cycles for non-compact spaces was discussed in Ref. [21]. In particular, the results [17] were used define the Jones-Witten polynomial 
for a dynamical system [22]. More recently the ideas from [18, 19, 20] were used to find new suitable higher dimensional generalizations of the asymptotic linking number starting from a topological BF theory (see [23] and references therein).

In the present paper we also use the notion of asymptotic $p$-cycles to extend the Donaldson-Witten and Seiberg-Witten invariants when smooth $p$-vector fields are incorporated globally on the underlying four-manifold. The asymptotic $p$-cycles associated to $p$-vectors on the manifolds define real homology $p$-cycles on these manifolds. They will constitute refined topological invariants of dynamical systems which distinguish the triplet $(M, \mathcal{F}, \mu)$, where $M$ is a four-manifold, $\mathcal{F}$ is the foliation (possibly singular) associated to a $p$-vector and $\mu$ is a transverse measure of $\mathcal{F}$ which is invariant under holonomy. Two triplets $\left(M_{1}, \mathcal{F}_{1}, \mu_{1}\right)$ and $\left(M_{2}, \mathcal{F}_{2}, \mu_{2}\right)$ are differentiably equivalent if there is a diffeomorphism from $M_{1}$ to $M_{2}$, which sends the leaves of $\mathcal{F}_{1}$ to the leaves of $\mathcal{M}_{2}$ and the push-forward of $\mu_{1}$ is $\mu_{2}$. Moreover these invariants will constitute a generalization of the Donaldson-Witten invariants for such triplets. For instance, one of the main results here is that our invariants will distinguish triplets: if two triplets $\left(M_{i}, \mathcal{F}_{i}, \mu_{i}\right)(i=1,2)$ have the property that the four-dimensional Donaldson-Witten invariants of $M_{1}$ and $M_{2}$ are equal but our invariants are different then the corresponding systems of flows on them are not differentiably equivalent.

On the other hand it is well known that Donaldson-Witten invariants can be interpreted in terms of the scattering amplitude (at zero momentum) of an axion with a NS5-brane in the heterotic string theory [24]. This paper would suggest a possible physical interpretation of our invariants involving flows in terms of an averaged propagation of a closed string in a target space described in terms of the moduli space of positions of a NS5-brane. That means, a "continuous" flux of closed strings (propagating in the transverse space to the worldvolume of the NS5 brane) giving rise to an asymptotic 2-cycle. The diffuseness of the asymptotic cycle is determined by a flow (or set of flows) in the target space given by some field in the target space, for instance, the NS $B$-field whose associated 2-vector field gives the 2-foliation on the target.

The organization of the present paper is as follows: Sec. 2 is devoted to a brief review of asymptotic $p$-cycles with $p>1$. In Sec. 3 we overview cohomological field theory for Donaldson-Witten theory. In Sec. 4 we define the Donaldson-Witten invariant for four-dimensional manifolds in the presence of a smooth and nowhere vanishing $p$-vector field over the underlying spacetime manifold. It is also verified that this invariant is well defined as a limiting average of the standard definition. Section 5 is devoted to describe the procedure for Kähler four-manifolds. This is done by using a physical procedure through the incorporation of a mass term which breaks the supersymmetry to $\mathcal{N}=1$ theories allowing the existence of a mass gap. In Section 6 we survey the Seiberg-Witten invariants. We focus mainly on the case of abelian magnetic monopoles. Non-abelian monopoles are also briefly described. In Sec. 7 we derive the Seiberg-Witten invariants in the presence of flows. Sec. 8 is devoted to explain how the Donaldson-Witten invariants for flows can be derived from a suitable system of strings in non-trivial flows on the spacetime target space. Finally, in Sec. 9 our final remarks and conclusions close the paper. 


\section{Asymptotic Cycles and Currents}

In this section we give a brief overview of asymptotic $p$-cycles with $p \geq 1$. Our aim is not to provide an extensive review of this material but introduce the notations and conventions of the relevant structures, which will be needed in the subsequent sections. For a more complete treatment see Refs. [17, 18, 19, 20, 25].

In order to study the main aim of the paper, which is a generalization of invariants of four-manifolds in the presence of a non-singular flows over a closed four-dimensional manifold $M$, it is necessary to consider asymptotic homology $p$-cycles of the flow on $M$ with values of $p$ greater than one. Here we will have two possibilities. The first one corresponds to a flow generated by a $p$-vector field which is not localized in the homology $p$-cycles of $M$. The second possibility is when the $p$-vector field is defined only on the tangent space of the $p$-cycles of $M$. Of course we could have a mixed situation. We also consider a set of flow invariant probability measures supported on the whole underlying manifold $M$. In this case the cycles constitute some "diffuse" cycles depending on the flow and the measure. The invariants constructed from these cycles detecting the differentiable structure of the four-manifolds with flows will be the asymptotic polynomial invariants of $M$. These invariants will coincide with the standard Donaldson-Witten invariants when the measure set is supported on the homology $p$-cycles $\gamma_{p}$ of $M$. For simply connected closed 4 -manifolds we will be interested in cycles of dimension $p=0,2,4$. From physical reasons $p=4$ is not an interesting case since it gives a topological term that can be added to the classical Lagrangian while that for $p=0$ it is a trivial cycle. Thus the only relevant cycle will be for $p=2$. In this section we define and interpret the observables as currents in terms of the winding number of asymptotic cycles.

The case $p=1$ was discussed in detail by Schwartzman in Ref. [17]. In Ref. [22] asymptotic cycles were applied to the Jones-Witten theory in order to find refined invariants of dynamical systems. Recently, these ideas were generalized to higher dimensions with foliations of dimension grater than one using the $B F$ theory without a cosmological constant in [23].

A current on a compact manifold $M$ of dimension $n$, is a linear and continuous functional in the de Rham complex $\Omega^{*}(M)$ i.e., satisfying:

$$
C\left[a_{1} \omega_{1}+a_{2} \omega_{2}\right]=a_{1} C\left[\omega_{1}\right]+a_{2} C\left[\omega_{2}\right],
$$

for all $\omega_{1}$ and $\omega_{2}$ differential forms and $a_{1}$ and $a_{2}$ scalars. As an example we define the following current $\gamma_{p}[\omega]=\int_{\gamma_{p}} \omega$, where $\gamma_{p}$ is a $p$-cycle of $M$ and $\omega$ is a $p$-form on $M$. Moreover a closed $(n-p)$-form $\alpha$ also defines a current in the following way

$$
\alpha[\omega]=\int_{M} \alpha \wedge \omega
$$

Another example is the contraction of a $p$-vector field $\nu_{p}$ and a $p$-form $\omega$. Let $\nu_{p}=$ $\nu^{i_{1} \ldots i_{p}} \partial_{i_{1}} \wedge \cdots \wedge \partial_{i_{p}}$ be a $p$-vector field and $\omega=\omega_{i_{1} \ldots i_{p}} d x^{i_{1}} \wedge \cdots \wedge d x^{i_{p}}$, then we have

$$
\nu_{p}[\omega]=\omega_{i_{1} \ldots i_{p}} \nu^{i_{1} \ldots i_{p}}
$$


A current restricted to the space of smooth $m$-forms is called an $m$-current. Let $\mathcal{D}_{m}$ denote the topological vector space (with the weak* topology ) of $m$-currents. Then in Ref. [25] there were constructed a series of boundary operators $\partial_{m}: \mathcal{D}_{m} \rightarrow \mathcal{D}_{m-1}$, defined on arbitrary $m$-currents, which define a chain complex and thus a homology theory which is dual to the de Rham cohomology.

\section{$2.1 \quad p$-Cycles and Geometrical Currents}

The definition of asymptotic cycles for higher dimensional foliations (under suitable hypothesis) starts by considering a closed subset $\mathcal{S}$ of a $n$-dimensional manifold $M$, a family of submanifolds $L_{\alpha}$ of dimension $p$, such that $\mathcal{S}=\cup_{\alpha} L_{\alpha}$ defines a partial foliation $\mathcal{F}_{p}$ (or lamination [26], see chapter 10) of dimension $p$. If $M$ is compact we cover all $M$ (including the interior) with a finite collection of closed disks $\mathbf{D}^{p} \times \mathbf{D}^{n-p}$ (horizontal and vertical disks respectively), these collections are called flow boxes and they are defined in such a way that they intersect each $L_{\alpha}$ in a set of horizontal disks $\left\{\mathbf{D}^{p} \times\{y\}\right\}$. The disks are smoothly embedded, such that the tangent planes vary continuously on the flow boxes.

A $(n-p)$ submanifold $T$ of $M$ is called a transversal if it is transversal to each $L_{\alpha}$ of the foliation $\mathcal{F}_{p}$. A transversal Borel measure of the foliation $\mathcal{F}_{p}$ assigns to each small $^{4}$ transversal submanifold $T$ a measure $\mu_{p, T}$. We assume that the measures are holonomy invariant and they are finite on compact subsets of the transversals [27].

Thus a geometrical current is the triple $\left(L_{\alpha}, \mu_{T}, \nu\right)$, with the entries being objects defined as above and $\nu$ is the orientation of $L_{\alpha}$, which is assigned to every point.

Assume that $M$ is covered by a system of flow boxes endowed with partitions of unity. Then every $p$-form $\omega$ can be decomposed into a finite sum $\omega=\sum_{i} \omega_{i}$, where each $\omega_{i}$ has his own support in the $i$-th flow box. We proceed to integrate out every $\omega_{i}$ over each horizontal disk $\left(\mathbf{D}^{p} \times\{y\}\right)_{i}$. Thus we obtain, using the transversal measure, a continuous function $f_{i}$ over $\left(\mathbf{D}^{n-p}\right)_{i}$, In this way we define a geometric current as

$$
\left\langle\left(L_{\alpha}, \mu_{T}, \nu\right), \omega\right\rangle=\sum_{i} \int_{\left(\mathbf{D}^{n-p}\right)_{i}} \mu_{T}(y)\left(\int_{\left(\mathbf{D}^{p} \times\{y\}\right)_{i}} \omega_{i}\right) .
$$

This current is closed in the sense of de Rham [25] i.e., if $\omega=d \phi$ where $\phi$ has compact support then $\left\langle\left(L_{\alpha}, \mu_{T}, \nu\right), d \phi\right\rangle=0$, since we can write $\phi=\sum_{i} \phi_{i}$. Ruelle and Sullivan [19] have shown that this current determines precisely an element of the $p$ th cohomology group of $M$. It does not depend of the choice of flow boxes. Recall that any $(n-p)$-form $\rho$ on $M$, determines a $p$-dimensional current by Poincaré duality $\langle\rho, \omega\rangle=\int_{M} \rho \wedge \omega$. Thus $(L, \mu, \nu)$ determines an element in $\operatorname{Hom}\left(H^{k}(M, \mathbb{R}), \mathbb{R}\right)$ which is isomorphic to $H_{k}(M, \mathbb{R})$ and therefore gives the asymptotic cycle.

Now consider an example of a geometrical current. Let $\mu_{p}$ be an invariant (under $\mathbf{X}_{p}$ ) volume $n$-form and $\mathbf{X}_{p}$ is a $p$-vector field nowhere vanishing on $M$. This defines a current in the de Rham sense via the $(n-p)$-form $\eta=i_{\mathbf{X}_{p}}\left(\mu_{p}\right)$. The current is given by

$$
W_{\mu, \mathbf{X}_{p}}(\beta)=\int_{M} i_{\mathbf{X}_{p}}\left(\mu_{p}\right) \wedge \beta .
$$

\footnotetext{
${ }^{4} \mathrm{~A}$ submanifold is said to be small if it is contained in a single flow box.
} 
This current is not in general closed but it will be closed, for instance, if the $p$-vector $\mathbf{X}_{p}$ consists of vector fields corresponding to one-parameter subgroups of an action of a Lie group which preserves the volume form $\mu_{p}$. More precisely, one can obtain asymptotic cycles for values of $p>1$ [18] as follows. Consider the action of a connected Lie group $G$ on a smooth compact oriented manifold $M$, whose orbits are of the same dimension $p$. A quantifier is a continuous field of $p$-vectors on $M$ everywhere tangent to the orbits and invariant under the action of $G$ via the differential.

A quantifier is said to be positive if it is nowhere vanishing and determines the orientation of the tangent space. A preferred action is an oriented action of a connected Lie group $G$ such that for any $x \in M$ the isotropy group $I_{x}$ of $x$ is a normal subgroup of $G$ and $G / I_{x}$ is unimodular.

In [18] it was proved that a preferred action possesses a positive quantifier and given a positive quantifier we can define a $1-1$ correspondence between finite invariant measures and transversal invariant measures. An important result which will be used in the next sections is the following theorem (Schwartzman [18]) that states: Let $\mathbf{X}_{p}$ be a positive definite quantifier (i.e. $p$-vector field) and $\mu_{p}$ an invariant measure given by a the volume $n$-form, then $i_{\mathbf{X}_{p}}\left(\mu_{p}\right)$ is a closed $(n-p)$-form and the asymptotic cycle $W_{\mu, \mathbf{X}_{p}}$ will be obtained by Poincaré duality of an element of $H^{n-p}(M, \mathbb{R})$ determined by $i_{\mathbf{X}_{p}}\left(\mu_{p}\right)$.

If $W_{\mu, \mathbf{x}_{p}}$ in $H_{p}(M, \mathbb{R})$ is an asymptotic cycle, the theorem gives an explicit way to construct asymptotic cycles and interpret currents as winding cycles, if the above conditions are satisfied. In [20] Sullivan gave another way of specifying a foliation, using structures of $p$-cones and operators acting over vectors on these cones.

One concrete example of the above is the following: Let $G$ be a connected abelian Lie group (for instance $\mathbb{R}^{n}$ or a compact torus $\mathbb{T}^{n}$ ) acting differentiably and locally freely (i.e. the isotropy group of every point is a discrete subgroup of $G$ ) on the smooth closed manifold $M$. Then, the orbits of the action determines a foliation with leaves of the same dimension as $G$. Since the group is abelian it has an invariant volume form and we obtain a natural foliated cycle.

\section{Overview of Cohomological Quantum Field The- ory: Donaldson-Witten Invariants}

In this section we overview briefly the Donaldson-Witten invariants for a closed, oriented and Riemannian four-manifold $M[3,4]$, representing our spacetime. We will focus on the Witten description [5] in terms of correlation functions (expectation values of some BRST-invariant operators). A cohomological field theory is a field theory with a BRST-like operator $\mathcal{Q}$ transforming as a scalar with respect to the spacetime symmetries. This operator represents a symmetry of the theory and it is constructed such that $\mathcal{Q}^{2}=0$. The Lagrangians of these theories can be written as a BRST commutator (BRST-exact) $L=\{\mathcal{Q}, V\}$, for some functional $V$. Given the properties of $\mathcal{Q}$, it implies that the Lagrangian is invariant under the $\mathcal{Q}$ symmetry $\{\mathcal{Q}, L\}=0$ i.e. the Lagrangian is $\mathcal{Q}$-closed. In general, all observables $\mathcal{O}$ in the theory are BRST invariant and they define cohomology classes given by $\mathcal{O} \sim \mathcal{O}+\{\mathcal{Q}, \lambda\}$ for some $\lambda$. Here $\mathcal{O}$ are 
the observables of the theory, which are invariant polynomials of the fields under the symmetry generator $\mathcal{Q}$. The observables are given by local field operators thus they depends on the point $x \in M$. Sometimes, in order to simplify the notation, we will omit explicitly this dependence.

Usually the relevant topological (twisted) Lagrangian can be derived from a physical Lagrangian which may depend on the Riemannian metric $g_{\mu \nu}$ of the underlying spacetime manifold $M$ and consequently there exists an energy-momentum tensor $T_{\mu \nu}$ which is also BRST-exact, i.e. $T_{\mu \nu}=\left\{\mathcal{Q}, \lambda_{\mu \nu}\right\}$, for some $\lambda_{\mu \nu}$. It was proved for any BRST-exact operator $\mathcal{O}$ that the correlation function $\langle\{\mathcal{Q}, \mathcal{O}\}\rangle$ vanishes and the partition function is also independent of the metric and the physical parameters encoded in the Lagrangian. Thus the correlation functions are topological invariants.

There are several examples of these kind of theories. In particular, the theories that we are interested in are the Donaldson-Witten and the Seiberg-Witten ones. From the physical point of view these theories come from a suitable twist to the Lorentz group of the $\mathcal{N}=2$ supersymmetric Yang-Mills theories with a compact Lie group (for definiteness we will use $S U(2)$ though its generalization to higher dimensional groups is not difficult). The supercharges also are affected by the twist which gives rise to our $\mathcal{Q}$ transforming as a scalar in the new assignation of the representations of spacetime global symmetries.

In the path integral formalism the Donaldson-Witten polynomials are given by correlation functions in the Euclidean signature

$$
\langle\mathcal{O}\rangle=\int \mathcal{D} \mathcal{X} \exp \left(-L_{D W} / e^{2}\right) \mathcal{O}
$$

where $L_{D W}$ is the Donaldson-Witten Lagrangian, $e$ is the coupling constant, the $\mathcal{D} \mathcal{X}$ represent the measure of the fields in the theory which includes a non-abelian gauge $A_{\mu}^{a}(x)$, scalar $\phi(x)$, fermionic $\psi(x)$ and ghost (anti-ghost) fields, all of them taking values in the adjoint representation of the gauge group. Fields $\left(A_{\mu}^{a}(x), \psi(x), \phi(x)\right)$ (with associated ghost numbers $U=(0,1,2)$ ) constitutes a fermionic BRST multiplet. We note that there is a nice mathematical interpretation of the mentioned ingredients of the theory. For instance, the fields will represent differential forms on the moduli space of the theory, the ghost number of the fields corresponds with the degree of these differential forms and the BRST charge $\mathcal{Q}$, which changes the ghost number in a unit, can be regarded as the exterior derivative.

It is possible to see that the change of the correlation functions with respect to the coupling constant $e$ is $\mathcal{Q}$-exact. Due to the property mentioned above $\langle\{\mathcal{Q}, V\}\rangle=0$, for some $V$, the correlation functions are independent of $e$. Consequently they can be computed in the semi-classical regime when $e$ is small and they can be evaluated by the stationary phase method. The path integration in the space $\mathcal{C}=\mathcal{B} / \mathcal{G}$ (of all gauge connections $\mathcal{B}$ modulo gauge transformations $\mathcal{G}$ ) localizes precisely in the space of gauge fields satisfying the instanton equation (anti-Self-dual Yang-Mills equations): $\widetilde{F}_{\mu \nu}=$ $-F_{\mu \nu}$ i.e. the instanton moduli space $\mathcal{M}_{D}$ of dimension $\left.d\left(\mathcal{M}_{D}\right)=8 p_{1}(E)-\frac{3}{2}(\chi+\sigma)\right)$ [28]. Here $\chi$ and $\sigma$ are the Euler characteristic and the signature of $M$ respectively.

It is worth mentioning that in general $\mathcal{M}_{D}$ has singularities which are associated with the reducible connections or the zero size instantons (small instantons). If one 
considers four manifolds with $b_{2}^{+}(M)>1$ the moduli space $\mathcal{M}_{D}$ behaves as an smooth, orientable and compact manifold $[3,4]$. But for general $b_{2}^{+}(M)$ it is more involved. Thus, in general, they are usually neglected by assuming that the only zero modes come from the gauge connection $A_{\mu}^{a}(x)$ and its BRST partner $\psi_{\mu}^{a}(x)$. Scalar field $\phi^{a}(x)$ has zero modes in the singularities and this would lead to a modification of the observables. The Donaldson map $H_{p}(M) \rightarrow H^{4-p}\left(\mathcal{M}_{D}\right)(p=0, \ldots, 4)$ is given by $\gamma_{p} \mapsto \int_{\gamma_{p}} c_{2}(\mathcal{P})$, where $\mathcal{P}$ is the universal bundle over $M \times \mathcal{M}_{D} \subset M \times \mathcal{B} / \mathcal{G}$ and $c_{2}$ is the second Chern class of $\mathcal{P}$.

For the gauge group $S U(2)$, the observables are

$$
\mathcal{O}^{\gamma_{p}} \equiv \int_{\gamma_{p}} W_{\gamma_{p}}
$$

where $\gamma_{p}$ is a $p$-homology cycle of $M$ and $W_{\gamma_{p}}$ is a $p$-form over $M$ given by

$$
\begin{array}{r}
W_{\gamma_{0}}(x)=\frac{1}{8 \pi^{2}} \operatorname{Tr} \phi^{2} \quad W_{\gamma_{1}}=\frac{1}{4 \pi^{2}} \operatorname{Tr}(\phi \wedge \psi), \\
W_{\gamma_{2}}=\frac{1}{4 \pi^{2}} \operatorname{Tr}(-i \psi \wedge \psi+\phi F), \quad W_{\gamma_{3}}=\frac{1}{4 \pi^{2}} \operatorname{Tr}(\psi \wedge F), \quad W_{\gamma_{4}}=\frac{1}{8 \pi^{2}} \operatorname{Tr}(F \wedge F),
\end{array}
$$

where $W_{\gamma_{0}}(x)$ is, by construction, a Lorentz and $\mathcal{Q}$ invariant operator. These observables have ghost number $U=(4,3,2,1,0)$ respectively and they are constructed as descendants which can be obtained from the relation

$$
d W_{\gamma_{p}}=\left\{\mathcal{Q}, W_{\gamma_{p+1}}\right\}
$$

This construction establishes an isomorphism between the BRST cohomology $H_{B R S T}^{*}(\mathcal{Q})$ and the de Rham cohomology $H_{d R}^{*}(M)$. One can check that $\mathcal{O}^{\gamma_{p}}$ is BRST-invariant (BRST-closed)

$$
\left\{\mathcal{Q}, \mathcal{O}^{\gamma_{p}}\right\}=\int_{\gamma_{p}}\left\{\mathcal{Q}, W_{\gamma_{p}}\right\}=\int_{\gamma_{p}} d W_{\gamma_{p-1}}=0 .
$$

For that reason the BRST commutator of $\mathcal{O}^{\gamma_{p}}$ only depends of the homology class of $\gamma_{p}$. Indeed, suppose that $\gamma_{p}=\partial \beta_{p+1}$, then we get (BRST-exact)

$$
\mathcal{O}^{\gamma_{p}}=\int_{\gamma_{p}} W_{\gamma_{p}}=\int_{\beta_{p+1}} d W_{\gamma_{p}}=\int_{\beta_{p+1}}\left\{\mathcal{Q}, W_{\gamma_{p+1}}\right\}=\left\{\mathcal{Q}, \int_{\beta_{p+1}} W_{\gamma_{p+1}}\right\} .
$$

Then the correlation functions are written as

$$
\begin{aligned}
\left\langle\mathcal{O}^{\gamma_{p_{1}}} \cdots \mathcal{O}^{\gamma_{p_{r}}}\right\rangle & =\left\langle\prod_{j=1}^{r} \int_{\gamma_{p_{j}}} W_{\gamma_{p_{j}}}\right\rangle \\
& =\int \mathcal{D} \mathcal{X} \exp \left(-L_{D W} / e^{2}\right) \prod_{j=1}^{r} \int_{\gamma_{p_{j}}} W_{\gamma_{p_{j}}} .
\end{aligned}
$$

These are the Donaldson-Witten invariants in the path integral representation. They are invariants of the smooth structure of $M$. For simply connected manifolds $\pi_{1}(M)=0$ the relevant cycles are of the dimensions $p=0,2$ and 4 . 
Consider a simply connected four-manifold $M$. The correlation functions of $r$ observables $\mathcal{O}_{\Sigma_{1}}, \ldots, \mathcal{O}_{\Sigma_{r}}$ is given by

$$
\begin{aligned}
\left\langle\mathcal{O}_{\Sigma_{1}}\left(x_{1}\right) \cdots \mathcal{O}_{\Sigma_{r}}\left(x_{r}\right)\right\rangle & =\left\langle\prod_{j=1}^{r} \mathcal{O}_{\Sigma_{j}}\left(x_{j}\right)\right\rangle \\
& =\int \mathcal{D} \mathcal{X} \exp \left(-L_{D W} / e^{2}\right) \prod_{j=1}^{r} \mathcal{O}_{\Sigma_{j}}\left(x_{j}\right),
\end{aligned}
$$

where we have considered only $r$ arbitrary 2-cycles i.e. $\gamma_{2_{j}}=\Sigma_{j}$ with $j=1, \ldots, r$. Since $\mathcal{O}_{\Sigma_{j}}\left(x_{j}\right)$ has ghost number $U=2$, the above correlation function has $U=2 r$. In terms of the zero modes one can write each $\mathcal{O}_{\Sigma_{j}}\left(x_{j}\right)=\Phi_{i_{1} i_{2}}\left(a_{i}\right) \psi^{i_{1}} \psi^{i_{2}}$, which absorbs two zero modes and consequently in the weak coupling limit we have

$$
\left\langle\mathcal{O}_{\Sigma_{1}}\left(x_{1}\right) \cdots \mathcal{O}_{\Sigma_{r}}\left(x_{r}\right)\right\rangle=\int_{\mathcal{M}_{D}} \Phi_{\Sigma_{1}} \wedge \cdots \wedge \Phi_{\Sigma_{r}} .
$$

Thus we have $\Sigma \in H_{2}(M) \rightarrow \Phi_{\Sigma} \in H^{2}\left(\mathcal{M}_{D}\right)$ and Eq.(14) becomes:

$$
\left\langle\mathcal{O}_{\Sigma_{1}}\left(x_{1}\right) \cdots \mathcal{O}_{\Sigma_{r}}\left(x_{r}\right)\right\rangle=\#\left(H_{\Sigma_{1}} \cap \cdots \cap H_{\Sigma_{r}}\right),
$$

where $H_{\Sigma_{j}}$ is the Poincaré dual to $\Phi_{\Sigma_{j}}$ and represents a $\left(d\left(\mathcal{M}_{D}\right)-2\right)$-homology cycle of the instanton moduli space $\mathcal{M}_{D}$. Equation (15) is interpreted as the intersection number of these homology cycles in the moduli space.

The topological invariance is not evident from the Eq. (12). However the above construction has a natural interpretation in terms of equivariant cohomology [29]. Moreover Atiyah and Jeffrey [30] showed that this expression can be reinterpreted in terms of the Euler class of a suitable infinite dimensional vector bundle in the Mathai-Quillen formalism [31]. This construction requires a real vector bundle $\mathcal{E}$ over the quotient space $\mathcal{C}$ of the space of all connections $\mathcal{B}$ modulo gauge transformations $\mathcal{G}$. This bundle is such that the fibres are the space of sections $\Gamma\left(\Lambda^{2,+} \otimes \operatorname{ad}(P)\right)$. Here $P$ is the $\mathrm{SU}(2)$-principal bundle over $M$ with gauge connection $A_{\mu}^{a}(x)$.

Moreover a section $s$ of $\mathcal{E}$ is given by $s=-F^{+}$, i.e. the locus $s^{-1}(0)$ is precisely the anti-self-dual moduli space $\mathcal{M}_{D} \subset \mathcal{C}$. The Euler class $e(\mathcal{E})$ is the pullback $s^{*} \Phi(\mathcal{E})$ of the Thom class $\Phi(\mathcal{E})$ of $\mathcal{E}$, under the section $s$. If $d(\mathcal{C})$ is the dimension of $\mathcal{C}$, the work of Mathai-Quillen [31] allows to gave a gaussian representative for the associated Thom class given by $e_{s, \nabla}(\mathcal{E})=\exp \left[-\frac{1}{e^{2}}|s|^{2}+\cdots\right]$, (which is given by a $2 m$ differentiable form on $\mathcal{C}$ ) such that the Euler class is given by

$$
\int_{\mathcal{C}} e_{s, \nabla}(\mathcal{E}) \wedge \alpha
$$

where $\alpha$ is an appropriate differential form of co-dimension $2 m$ i.e. $\alpha \in H^{d(\mathcal{C})-2 m}(\mathcal{C})$. This Euler class is of course independent on the connection $\nabla$ used in this construction. Euler class (16), for an appropriate $\alpha$, represents the Donaldson-Witten invariants (12). 


\section{Donaldson-Witten Invariants for Flows}

In this section we study the Donaldson-Witten invariants when there exist flows associated to a $p$-vector field over the spacetime manifold $M$ equipped with an invariant probability measure $\mu$ (normalized such that $\int_{M} \mu=1$ ) and a non-singular $p$-vector field $\mathbf{Y}_{p}=Y_{1} \wedge \cdots \wedge Y_{p}$ where $Y_{i}=Y_{i}^{\mu} \partial_{\mu}$ with $\mu=1, \ldots, 4$ and $i=1, \cdots, p$. We require that the probability measure $\mu_{T, p}$ be invariant under $\mathbf{Y}_{p}$ for every $p$. Thus, the global information is encoded in the set of triplets $\left\{\left(M, \mathcal{F}_{p}, \mu_{T, p}\right), p=0, \ldots, 4\right\}$, where $\mathcal{F}_{p}$ is the foliation generated by the $p$-vector field $\mathbf{Y}_{p}$. Each triplet $\left(M, \mathcal{F}_{p}, \mu_{T, p}\right)$ determines an asymptotic $p$-cycle that we denote as $\widetilde{\gamma}_{p}$.

Now we define the generalized Lie derivative $\mathcal{L}_{\mathbf{Y}_{p}}$ for $p$-vectors which is a graded operator defined as follows

$$
\mathcal{L}_{\mathbf{Y}_{p}} \omega=\left[d, i_{\mathbf{Y}_{p}}\right] \omega=d\left(i_{\mathbf{Y}_{p}} \omega\right)+(-1)^{\operatorname{deg} d \cdot \operatorname{deg} i_{Y}} i_{\mathbf{Y}_{p}}(d \omega),
$$

being $\left[d, i_{\mathbf{Y}_{p}}\right]$ a graded commutator and $\omega$ is a $p$-form. For further details on the formalism of multi-vector field see $[32,33]$. Here there are two possibilities:

- The homology groups associated to the orbits of the $p$-vector fields $\mathbf{Y}_{p}$ 's are trivial therefore they will not give relevant information of the four-manifold, however we can use these trivial asymptotic homology cycles to describe some particular interesting configurations of flows. This case corresponds to the situations found in Refs. [22, 23]. In particular if $\omega$ is the volume form invariant under $\mathbf{Y}_{p}$ for every $p$, the last term of the previous equation is zero, then the Lie derivative $\mathcal{L}_{\mathbf{Y}_{p}} \mu_{p}=d\left(i_{\mathbf{Y}_{p}} \mu_{p}\right)$, the term $i_{\mathbf{Y}_{p}} \mu_{p}$ looks like the expression from the Schwartzman theorem at the end of Sec. 2. In order for $i_{\mathbf{Y}_{p}} \mu_{p}$ to be a cohomology class it needs to be closed. This requirement is established by the following equation

$$
\mathcal{L}_{\mathbf{Y}_{p}} \mu_{p}=0
$$

If this condition implies $d\left(i_{\mathbf{Y}_{p}} \mu_{p}\right)=0$ i.e. $i_{\mathbf{Y}_{p}} \mu_{p}$ is closed then this element defines an element of the $(n-p)$ cohomology group.

- The other possibility corresponds to the case when the $\widetilde{\gamma}_{p}$ are orbits of the flow generated by the $p$-vector fields $\mathbf{Y}_{p}$ for each value of $p$. These cycles are nontrivial. In this case it gives rise to a generalization of the four-manifold invariants as the measure $\mu$ is supported in the whole manifold $M$.

In the present paper we will focus mainly on this second possibility.

\subsection{The Definition of Observables}

Now we will introduce flows over $M$, and promote its homology cycles to asymptotic cycles. We define the asymptotic observable for a $p$-vector fields $(p=1, \ldots, 4)$ according to the expression

$$
\widetilde{\mathcal{O}}_{\mathbf{Y}_{p}}\left(\mu_{p}\right)=\int_{\widetilde{\gamma}_{p}} W_{\gamma_{p}}:=\int_{M} i_{\mathbf{Y}_{p}}\left(W_{\gamma_{p}}\right) \mu_{p}(x),
$$


where $\mu_{p}$ is the volume form of $M$ invariant under $\mathbf{Y}_{p}$ and $i_{\mathbf{Y}_{p}}\left(W_{\gamma_{p}}\right)$ denotes the contraction and $\mathrm{Tr}$ is the trace of the adjoint representation of the gauge group.

We can think of the observable as an average winding number of the asymptotic cycle. The observables are related to the asymptotic cycles then they carry information about the flow whether it is trivial or not.

Let $\mathbf{Y}_{1}, \ldots, \mathbf{Y}_{4}$ be $p$-vector fields $(p=1, \ldots, 4)$ and together with the expressions (7) and (8) we define the asymptotic observable as

$$
\begin{array}{r}
\widetilde{\mathcal{O}}_{\mathbf{Y}_{0}}\left(\mu_{0}\right) \equiv \mathcal{O}^{\gamma_{0}}(x)=\frac{1}{8 \pi^{2}} \operatorname{Tr} \phi^{2}, \\
\widetilde{\mathcal{O}}_{\mathbf{Y}_{1}}\left(\mu_{1}\right)=\int_{M} \operatorname{Tr} \frac{1}{4 \pi^{2}} i_{\mathbf{Y}_{1}}(\phi \psi) \mu_{1}, \\
\widetilde{\mathcal{O}}_{\mathbf{Y}_{2}}\left(\mu_{2}\right)=\int_{M} \operatorname{Tr} \frac{1}{4 \pi^{2}} i_{\mathbf{Y}_{2}}(-i \psi \wedge \psi+\phi F) \mu_{2}, \\
\widetilde{\mathcal{O}}_{\mathbf{Y}_{3}}\left(\mu_{3}\right)=\int_{M} \operatorname{Tr} \frac{1}{4 \pi^{2}} i_{\mathbf{Y}_{3}}(\psi \wedge F) \mu_{3}, \\
\widetilde{\mathcal{O}}_{\mathbf{Y}_{4}}\left(\mu_{4}\right)=\int_{M} \operatorname{Tr} \frac{1}{8 \pi^{2}} i_{\mathbf{Y}_{4}}(F \wedge F) \mu_{4} .
\end{array}
$$

It is an easy matter to check that these asymptotic observables $\widetilde{\mathcal{O}}_{\mathbf{Y}_{p}}\left(\mu_{p}\right)$ are $\mathcal{Q}$ invariant

$$
\begin{aligned}
\left\{\mathcal{Q}, \widetilde{\mathcal{O}}_{\mathbf{Y}_{p+1}}\left(\mu_{p+1}\right)\right\} & =\left\{\mathcal{Q}, \int_{M} i_{\mathbf{Y}_{p+1}}\left(W_{\gamma_{p+1}}\right) \mu_{p+1}\right\} \\
& =\int_{M}\left\{\mathcal{Q}, i_{\mathbf{Y}_{p+1}}\left(W_{\gamma_{p+1}}\right)\right\} \mu_{p+1} \\
& =\int_{M} i_{\mathbf{Y}_{p+1}}\left(d W_{\gamma_{p}}\right) \mu_{p+1}=0 .
\end{aligned}
$$

Here we have used the fact that the measure is invariant under the flow i.e. these observables are closed in the de Rham sense (see theorem 2A from Ref. [18]) and the fact that the BRST charge $\mathcal{Q}$ commutes with the contraction operation $i_{\mathbf{Y}_{p}}$. Then these asymptotic observables are BRST invariant, therefore they will give rise to topological invariants of dynamical system through a generalization of the Donaldson-Witten invariants.

Moreover we observe that the problem arising in the Jones-Witten case [22], which distinguishes strongly the abelian and non-abelian cases is absent here and for the present case, the non-abelian case can be treated exhaustively. Even if the theory is non-abelian, our observables are Lie algebra-valued $p$-forms and the group and spacetime information decouples. For the gauge group $\mathrm{SU}(N)$ with Lie algebra $\mathrm{su}(N)$ we take for instance

$$
\begin{gathered}
\widetilde{\mathcal{O}}_{\mathbf{Y}_{2}}\left(\mu_{2}\right)=\int_{M} \frac{1}{4 \pi^{2}} \operatorname{Tr} i_{\mathbf{Y}_{2}}(-i \psi \wedge \psi+\phi F) \mu_{2} \\
=\int_{M} \frac{1}{4 \pi^{2}} \operatorname{Tr}\left\{i_{\mathbf{Y}_{2}}\left(-i \psi^{a} \wedge \psi^{b}+\phi^{a} F^{b}\right) t_{a} t_{b}\right\} \mu_{2},
\end{gathered}
$$


where if $t_{a}$ and $t_{b}$ are generators of $\mathrm{su}(2)$ they satisfy the normalization condition: $\operatorname{Tr}\left(t_{a} t_{b}\right)=\frac{1}{2} \delta_{a b}$. Then last expression takes the following form

$$
\widetilde{\mathcal{O}}_{\mathbf{Y}_{2}}\left(\mu_{2}\right)=\int_{M} \frac{1}{8 \pi^{2}} i_{\mathbf{Y}_{2}}\left(-i \psi^{a} \wedge \psi^{a}+\phi^{a} F^{a}\right) \mu_{2}
$$

We will use the following notation for $r$ components of $p$-cycles of different dimension. The observables will be denoted by $\widetilde{\mathcal{O}}_{\mathbf{Y}_{p_{j}}}\left(\mu_{p_{j}}\right)$, where $p_{j}$ take values $1, \ldots, 4, j=1, \ldots, r$ and such that they satisfy $\sum_{p, j} p_{j}=\mathrm{d}\left(\mathcal{M}_{\mathrm{D}}\right)$, which is the dimension of the moduli space of instantons.

\subsection{Donaldson-Witten Invariants of four-manifolds for Flows}

For an oriented manifold $M$ with $p_{j}$-vectors fields $\mathbf{Y}_{p_{j}}$, with probability invariant measure $\mu_{p_{j}}$, the $r$-point correlation functions (Donaldson-Witten invariants) for flows $\mathbf{Y}_{p_{j}}$ is given by

$$
\left\langle\widetilde{\mathcal{O}}_{\mathbf{Y}_{p_{1}}}\left(\mu_{p_{1}}\right) \cdots \widetilde{\mathcal{O}}_{\mathbf{Y}_{p_{r}}}\left(\mu_{p_{r}}\right)\right\rangle=\int \mathcal{D} \mathcal{X} \exp \left(-L_{D W} / e^{2}\right) \prod_{j=1}^{r} \int_{M} i_{\mathbf{Y}_{p_{j}}}\left(W_{\gamma_{p_{j}}}\right) \mu_{p_{j}}(x) .
$$

This expression is reduced to the ordinary Donaldson-Witten invariants (12), when the measure is supported on the cycles. One can think of this set of measures $\left\{\mu_{p_{j}}\right\}$ as Dirac measures on the set of $p-j$-cycles $\left\{\widetilde{\gamma}_{p_{j}}\right\}$. If we consider the invariant probability measure $\mu_{p}=\sum_{j}^{r} \mu_{p_{j}}$, where each $\mu_{p_{j}}$ is supported on $\widetilde{\gamma}_{p_{j}}$ and they are uniformly distributed with respect the coordinates of $\left\{\widetilde{\gamma}_{p_{j}}\right\}$. In other words $\mu_{p_{j}}$ is supported on $\gamma_{p_{j}}$ and it coincides with the normalized area form of the surface $\gamma_{p_{j}}$. We need to normalize in order to have $\mu$ a probability measure.

We want to remark that the underlying $p_{j}$-fields $\mathbf{Y}_{p_{j}}$ will be considered here just as spectator fields. That is, they are background fields that are not of dynamical nature and don't represent additional degrees of freedom of the underlying theory. Thus, they don't contribute to the measure $\mathcal{D} \mathcal{X}$, to the Lagrangian $L_{D W}$ nor to the counting of zero modes and consequently they do not lead to a modification of the dimension of the moduli space of instantons ${ }^{5}$. There will be an influence of these $p_{j}$-vector fields to our systems modifying mainly the structure of the observables of the theory. The structure of the vacuum also remains unchanged i.e. the mass gap and the chiral symmetry breaking are still playing an important role in the definition of invariants.

For the moment we will consider an arbitrary operator $\widetilde{\mathcal{O}}_{\mathbf{Y}_{p_{j}}}\left(\mu_{p_{j}}\right)$ with $p_{j} \geq 1$ (because in the case $p_{j}=0$ there is not a flow). Now we proceed to perform the integral over the non-zero modes, as in the case without flows.

We assume that the only zero modes correspond to the gauge field $A_{\mu}$ and those associated to $\psi_{\mu}$. Denote this observable by $\widetilde{\mathcal{O}}_{\mathbf{Y}_{p_{j}}}\left(\mu_{p_{j}}\right)=\widetilde{\Phi}_{i_{1} \cdots i_{n}}\left(a_{i}, \mathbf{Y}_{p_{j}}\right) \psi^{i_{1}} \ldots \psi^{i_{n}}$,

\footnotetext{
${ }^{5}$ Thus we compute the effect of these spectator vector fields on the invariants. At this stage it is not possible to compute the back reaction of all dynamical fields to $\mathbf{Y}_{p_{j}}$.
} 
where $a$ 's denotes the zero modes of the gauge field and $\psi$ 's are the zero modes of the fermionic field, $\widetilde{\Phi}\left(a_{i}, \mathbf{Y}_{p_{j}}\right)$ is a function that only depends on the zero modes of the gauge field and contains the information about the flow. As in the standard case the partition function is zero, the integrals which are non-zero are of the form (28), where $\widetilde{\mathcal{O}}$ absorb the zero modes.

Performing the functional integration over the non-zero modes in the weak coupling limit we get $\widetilde{\Phi}_{i_{1} \cdots i_{n}}\left(a_{i}, \mathbf{Y}_{p_{j}}\right)$ is an skew-symmetric tensor then $\widetilde{\mathcal{O}}$ can be regarded as a $n=d\left(\mathcal{M}_{D}\right)$-form in $\mathcal{M}_{D}$. Consequently the correlation functions of one observable $\widetilde{\mathcal{O}}$ reads

$$
\begin{aligned}
\left\langle\widetilde{\mathcal{O}}_{\mathbf{Y}_{p_{j}}}\left(\mu_{p_{j}}\right)\right\rangle & =\int_{\mathcal{M}_{D}} d a_{1} \ldots d a_{n} d \psi^{1} \ldots d \psi^{n} \widetilde{\Phi}_{i_{1} \ldots i_{n}}\left(a_{i}, \mathbf{Y}_{p_{j}}\right) \psi^{i_{1}} \ldots \psi^{i_{n}} \\
& =\int_{\mathcal{M}_{D}} \widetilde{\Phi}_{\mathbf{Y}_{d\left(\mathcal{M}_{D}\right)}},
\end{aligned}
$$

where we integrate out the $a_{i}$ 's and obtain a $n$-form $\widetilde{\Phi}$ defined in the moduli space $\mathcal{M}_{D}$. If one considers a product of observables $\widetilde{\mathcal{O}}=\widetilde{\mathcal{O}}_{\mathbf{Y}_{p_{1}}}\left(\mu_{p_{1}}\right) \cdots \widetilde{\mathcal{O}}_{\mathbf{Y}_{p_{r}}}\left(\mu_{p_{r}}\right)$ with $\sum_{p, j} p_{j}=n=d\left(\mathcal{M}_{D}\right)$ and $p_{j}$ being the number of zero modes of $\widetilde{\mathcal{O}}_{\mathbf{Y}_{p_{j}}}\left(\mu_{p_{j}}\right)$ then, in analogy to Ref. [23] one obtains:

$$
\left\langle\widetilde{\mathcal{O}}_{\mathbf{Y}_{p_{1}}}\left(\mu_{p_{1}}\right) \cdots \widetilde{\mathcal{O}}_{\mathbf{Y}_{p_{r}}}\left(\mu_{p_{r}}\right)\right\rangle=\int_{\mathcal{M}_{D}} \widetilde{\Phi}_{\mathbf{Y}_{p_{1}}} \wedge \cdots \wedge \widetilde{\Phi}_{\mathbf{Y}_{p_{r}}}
$$

These correlation functions are the asymptotic Donaldson-Witten invariants and they are invariants of the triplet $(M, \mathcal{F}, \mu)$. In order to compute the observables we integrate out the zero modes. This is completely analogous to the case without flows because the measure of the path integral does not include the $\mathbf{Y}^{\prime} s$

$$
\begin{array}{r}
\widetilde{\Phi}_{\mathbf{Y}_{0}}=\frac{1}{8 \pi^{2}} \operatorname{Tr}\langle\phi\rangle^{2}, \\
\widetilde{\Phi}_{\mathbf{Y}_{1}}=\int_{M} \operatorname{Tr} \frac{1}{4 \pi^{2}} i_{\mathbf{Y}_{1}}(\langle\phi\rangle \psi) \mu_{1}, \\
\widetilde{\Phi}_{\mathbf{Y}_{2}}=\int_{M} \operatorname{Tr} \frac{1}{4 \pi^{2}} i_{\mathbf{Y}_{2}}(-i \psi \wedge \psi+\langle\phi\rangle F) \mu_{2}, \\
\widetilde{\Phi}_{\mathbf{Y}_{3}}=\int_{M} \operatorname{Tr} \frac{1}{4 \pi^{2}} i_{\mathbf{Y}_{3}}(\psi \wedge F) \mu_{3}, \\
\widetilde{\Phi}_{\mathbf{Y}_{4}}=\int_{M} \operatorname{Tr} \frac{1}{8 \pi^{2}} i_{\mathbf{Y}_{4}}(F \wedge F) \mu_{4} .
\end{array}
$$

Now we define the intersection number in a way analogous to the case without flows.

For the simply connected case $\left(\pi_{1}(M)=0\right)$, we have that the important observables are those associated with cycles of dimension 0,2 and $4^{6}$. In general a $p_{j}$-cycle has associated an operator (form) with ghost number $U=4-p_{j}$, corresponding to the

\footnotetext{
${ }^{6} 0$ and 4 cycles are related by Hodge duality, so we will consider one of them, say 0-cycles.
} 
Donaldson map $\mu_{D}: H_{p}(M) \rightarrow H^{4-p}\left(\mathcal{M}_{D}\right)$. In Ref. [5] Witten constructed this map, interpreted as intersection number of cycles in the four manifold $M$

$$
\begin{aligned}
\left\langle\widetilde{I}_{\mathbf{Y}_{2_{1}}}\left(\mu_{2_{1}}\right)\left(x_{1}\right) \cdots \widetilde{I}_{\mathbf{Y}_{2_{r}}}\left(\mu_{2_{r}}\right)\left(x_{r}\right)\right\rangle & =\int_{\mathcal{M}_{D}} \nu_{\mathbf{Y}_{2_{1}}} \wedge \cdots \wedge \nu_{\mathbf{Y}_{2_{r}}} \\
& =\#\left(H_{\widetilde{\Sigma}_{\mathbf{Y}_{2_{1}}}} \cap \cdots \cap H_{\widetilde{\Sigma}_{\mathbf{Y}_{2 r}}}\right),
\end{aligned}
$$

where $H_{\widetilde{\Sigma}_{\mathbf{Y}_{2}}}$ is the Poincaré dual of codimension 2. If the observables $\widetilde{I}_{\mathbf{Y}_{2_{j}}}$ are denoted by $\widetilde{I}_{\mathbf{Y}_{j}}$ and $\mu_{2_{j}}$ is denoted by $\mu_{j}$, then the equation (36) represents the asymptotic intersection linking numbers of the 2 -flows in the moduli space $\mathcal{M}_{D}$ determined by the integration of differential two-forms $\nu_{j}$ 's on $\mathcal{M}_{D}$ depending on the set of 2 -vector fields $\left\{\mathbf{Y}_{j}\right\}_{j=1, \ldots, r}$ with $r=d / 2$. In terms of the asymptotic cycles Eq. (36) represents the asymptotic intersection number of $r$ asymptotic homology 2-cycles $\widetilde{\Sigma}_{\mathbf{Y}_{j}}$ in $M$.

Donaldson-Witten invariants $(12)$ are defined for $b_{2}^{+}(M)>1$. It is very interesting to know what is the analog condition for defining the existence of the corresponding asymptotic invariants for foliations. The analog of the wall-crossing that does exist in the Donaldson case for $b_{2}^{+}(M)=1$, will be also of interest in the context of foliations. We leave this question for future work.

\subsection{Asymptotic Intersection Numbers}

In this subsection we use the dynamics of strongly coupled supersymmetric gauge theories. In particular we use some features as: the existence of a mass gap, the cluster decomposition and a structure of the vacua degeneracy consisting of a finite number of discrete states obtained after a chiral symmetry breaking due to gaugino condensation. We proceed to find an interpretation of the intersection number for asymptotic cycles described in Eq. (36) with the aid of the mentioned features. In order to do that we are going to compute the 2-point correlation function of a pair of the observables $\widetilde{I}_{\mathbf{Y}_{1}}(\mu)$ at different points

$$
\left\langle\widetilde{I}_{\mathbf{Y}_{1}}\left(\mu_{1}\right)\left(x_{1}\right) \widetilde{I}_{\mathbf{Y}_{2}}\left(\mu_{2}\right)\left(x_{2}\right)\right\rangle=\#\left(H_{\widetilde{\Sigma}_{\mathbf{Y}_{1}}} \cap H_{\widetilde{\Sigma}_{\mathbf{Y}_{2}}}\right),
$$

where the $x_{i}$ 's are points on $M$ and $\#\left(H_{\widetilde{\Sigma}_{\mathbf{Y}_{1}}} \cap H_{\widetilde{\Sigma}_{\mathbf{Y}_{2}}}\right)$ represents the asymptotic intersection number of the asymptotic cycles $\widetilde{\Sigma}_{\mathbf{Y}_{1}}$ and $\widetilde{\Sigma}_{\mathbf{Y}_{2}}$.

As in the standard case without flows the one point correlation function $\left\langle\widetilde{I}_{\mathbf{Y}}(\mu)(x)\right\rangle$ is also zero, for the same reason that in the standard case. In $M=\mathbb{R}^{4}$ it vanishes by Lorentz invariance with the measure invariant under the flow, this yields

$$
\begin{gathered}
\left\langle\widetilde{I}_{\mathbf{Y}}(\mu)(x)\right\rangle=\int_{\widetilde{\Sigma}} d \sigma^{m n}\left\langle Z_{m n}\right\rangle \\
=\int_{M}\left\langle i_{\mathbf{Y}}(Z)\right\rangle \mu=\int_{M}\left\langle Y^{m n} Z_{m n}\right\rangle \mu .
\end{gathered}
$$


As the $\mathbf{Y}^{\prime}$ S are not dynamical fields their expectation values is given by $Y^{m n}\left\langle Z_{m n}\right\rangle$ and as $\left\langle Z_{m n}\right\rangle$ vanishes by Lorentz invariance then consequently $\left\langle\widetilde{I}_{\mathbf{Y}}(\mu)(x)\right\rangle$ also vanishes in flat spacetime. However for a general four-manifold in a theory with a mass gap (it is known that $\mathcal{N}=2$ gauge field theories in four dimensions don't have a mass gap, however we assume, following Witten [9], that is indeed the case ${ }^{7}$ ), the expectation value of the operator $Z_{m n} Y^{m n}$ is expanded, as in [9] in terms of local invariants of the Riemannian geometry of $M$

$$
\left\langle Z_{m n}(x) Y^{m n}(x)\right\rangle=D_{m} R D_{n} D_{s} D^{s} R \cdot Y^{m n} \pm \cdots
$$

Under the metric scaling $g \rightarrow t g$ with $t$ positive, the volume form $\mu$ scales as $t^{4}$, then $\left\langle Z_{m n}(x) Y^{m n}(x)\right\rangle$ should scale faster than $1 / t^{4}$. This is precisely achieved by the mass in the case we have flows. Thus, in general $\left\langle\int_{M} i_{\mathbf{Y}}(Z) \mu\right\rangle$ vanishes as $t \rightarrow \infty$.

Now we want to compute

$$
\left\langle\widetilde{I}_{\mathbf{Y}_{1}}\left(\mu_{1}\right)\left(x_{1}\right) \widetilde{I}_{\mathbf{Y}_{2}}\left(\mu_{2}\right)\left(x_{2}\right)\right\rangle=\int_{M_{1} \times M_{2}} G_{\mathbf{Y}_{1}, \mathbf{Y}_{2}}\left(x_{1}, x_{2}\right) \mu_{1}\left(x_{1}\right) \mu_{2}\left(x_{2}\right),
$$

where

$$
G_{\mathbf{Y}_{1}, \mathbf{Y}_{2}}\left(x_{1}, x_{2}\right)=\left\langle i_{\mathbf{Y}_{1}}(Z)\left(x_{1}\right) \cdot i_{\mathbf{Y}_{2}}(Z)\left(x_{2}\right)\right\rangle .
$$

Considering the properties of $i_{X_{1} \wedge \cdots \wedge X_{p}}$, one can see that the next formula holds

$$
i_{X_{1} \wedge \cdots \wedge X_{p}} B_{p} \wedge \mu_{n}-(-1)^{\frac{p}{2}(3+p)} B_{p} \wedge i_{X_{p} \wedge \cdots \wedge X_{1}} \mu_{n}=0
$$

where $B_{p}$ is any $p$-form. After some work it is easy to see that using the previous equation we have

$$
\left\langle\widetilde{I}_{\mathbf{Y}_{1}}\left(\mu_{1}\right)\left(x_{1}\right) \widetilde{I}_{\mathbf{Y}_{2}}\left(\mu_{2}\right)\left(x_{2}\right)\right\rangle=\int_{M_{1} \times M_{2}}\left(\Theta_{\mathbf{Y}_{1}} \wedge Z\right)\left(x_{1}\right) \wedge\left(\Theta_{\mathbf{Y}_{2}} \wedge Z\right)\left(x_{2}\right) \cdot \delta\left(x_{1}-x_{2}\right)
$$

where $\Theta_{\mathbf{Y}_{1}}=i_{\mathbf{Y}_{1}}\left(\mu_{1}\right)$ and $\Theta_{\mathbf{Y}_{2}}=i_{\mathbf{Y}_{2}}\left(\mu_{2}\right)$ are the Poincaré dual to $\widetilde{\Sigma}_{1}$ and $\widetilde{\Sigma}_{2}$ respectively. This is a double form [25] and consequently $G_{\mathbf{Y}_{1}, \mathbf{Y}_{2}}\left(x_{1}, x_{2}\right)$ is proportional to $\delta\left(x_{1}-x_{2}\right)$ and the only non-vanishing contributions come from the points $x_{1}=x_{2}$.

Equivalently one can follow a dimensional analysis with $g \rightarrow t g$, with $t \rightarrow \infty$. For $x_{1} \neq x_{2},\left\langle i_{\mathbf{Y}_{1}}\left(Z\left(x_{1}\right)\right) i_{\mathbf{Y}_{2}}\left(Z\left(x_{2}\right)\right)\right\rangle$ vanishes faster than $1 / t^{8}$. The only possible nonvanishing contribution is localized around $x_{1}=x_{2}$ as $t \rightarrow \infty$. These are precisely the intersection points of the asymptotic cycles. That is reduced to the transversely intersection of the flows in finitely many points. Thus we have

$$
\left\langle\widetilde{I}_{\mathbf{Y}_{1}}\left(\mu_{1}\right)\left(x_{1}\right) \widetilde{I}_{\mathbf{Y}_{2}}\left(\mu_{2}\right)\left(x_{2}\right)\right\rangle=\eta \cdot \#\left(\widetilde{\Sigma}_{\mathbf{Y}_{1}} \cap \widetilde{\Sigma}_{\mathbf{Y}_{2}}\right) \cdot\langle 1\rangle,
$$

\footnotetext{
${ }^{7}$ In the process of obtaining invariants of smooth manifolds from physical theories, the dynamics of these theories is an important guide. However the topological invariants are independent on the metric and the coupling constant one can compute these invariants in the limit where the theory is under control. The assumption of a mass gap for $\mathcal{N}=2$ theories is justified as it allows to compute invariants for some four-manifolds. However it is observed that the own theory tell us that one has to consider the full dynamics (including the supersymmetry breaking) in order to find the right invariants.
} 
where $\eta$ is a constant, $\langle 1\rangle=\exp (a \chi(M)+b \sigma(M))$ with $a, b$ being constants and $\chi(M)$ and $\sigma(M)$ the Euler characteristic and signature of $M$ respectively. In analogy with the definition of asymptotic linking number we define the asymptotic intersection number of two 2-flows generated by the 2 -vector fields $\mathbf{Y}_{1}$ and $\mathbf{Y}_{2}$. Thus $\left\langle\widetilde{I}_{\mathbf{Y}_{1}}\left(\mu_{1}\right)\left(x_{1}\right) \widetilde{I}_{\mathbf{Y}_{2}}\left(\mu_{2}\right)\left(x_{2}\right)\right\rangle$ can be interpreted as the average intersection number.

Let $\left\{\widetilde{\Sigma}_{\mathbf{Y}_{1}}, \ldots, \widetilde{\Sigma}_{\mathbf{Y}_{r}}\right\}$ be a set of $r$ arbitrary asymptotic homology 2-cycles. With the aid of cluster decomposition property for a vacua consisting of only one state, Eq. (44) can be used to write the generating functional of the correlation functions of observables associated to $r$ 2-cycles

$$
\left\langle\exp \left(\sum_{a} \alpha_{a} \widetilde{I}_{\mathbf{Y}_{a}}\left(\mu_{a}\right)\right)\right\rangle=\exp \left(\frac{\eta}{2} \sum_{a, b} \alpha_{a} \alpha_{b} \#\left(\widetilde{\Sigma}_{\mathbf{Y}_{a}} \cap \widetilde{\Sigma}_{\mathbf{Y}_{b}}\right)\right) \cdot\langle 1\rangle .
$$

This is given in terms of the pairwise intersection between the corresponding asymptotic cycles. If one incorporates the operators $\widetilde{\mathcal{O}}$ and take into account that the vacua consist of a finite set $\mathcal{S}$ of discrete states it can be modified as follows

$$
\left\langle\exp \left(\sum_{a} \alpha_{a} \widetilde{I}_{\mathbf{Y}_{a}}\left(\mu_{a}\right)+\lambda \widetilde{\mathcal{O}}\right)\right\rangle=\sum_{\rho \in \mathcal{S}} C_{\rho} \exp \left(\frac{\eta_{\rho}}{2} \sum_{a, b} \alpha_{a} \alpha_{b} \#\left(\widetilde{\Sigma}_{\mathbf{Y}_{a}} \cap \widetilde{\Sigma}_{\mathbf{Y}_{b}}\right)+\lambda\langle\mathcal{O}\rangle_{\rho}\right),
$$

where $C_{\rho}$ is a constant including the gravitational contribution of the curvature invariants $\chi(M)$ and $\sigma(M)$ coming from $<1>$. When the gauge group is $\mathrm{SU}(2)$ the chiral symmetry breaking tells that the set $\mathcal{S}$ is precisely $\mathbb{Z}_{2}$. Thus the above formula consists of two terms.

\section{Donaldson-Witten Invariants for Kähler Mani- folds with Flows}

In this section we discuss the Donaldson invariants on a Kähler 4-manifold $M$. We follow closely Ref. [9], from where we take the notation and conventions. We use the dynamics of strong coupling $\mathcal{N}=1$ supersymmetric gauge theories in four dimensions in the infrared. In particular, the perturbation of the $\mathcal{N}=2$ theory by adding a mass term $^{8}$ breaks supersymmetry leaving a theory with a remnant $\mathcal{N}=1$ supersymmetry. As we mentioned before, the properties of strong coupled gauge theories (confinement, mass gap and chiral symmetry breaking) are an important subject in order to compute the invariants of Kähler manifolds admitting a non-trivial canonical class in $H^{(2,0)}(M) \neq 0{ }^{9}$. We consider in addition a series of non-singular smooth flows generated by 2-vector fields $\mathbf{X}$ and $\mathbf{Y}$ over $M$. We want to describe how Donaldson-Witten invariants of Kähler manifolds will be modified in the presence of these flows ${ }^{10}$.

\footnotetext{
${ }^{8}$ The mass term consist of a quadratic term of a scalar superfield in the adjoint representation of the gauge group.

${ }^{9}$ The condition of the existence of a canonical class is related to $b_{2}^{+}(M)$ by $b_{2}^{+}(M)=$ $2 \operatorname{dim} H^{(2,0)}(M)+1$. Thus, the condition $H^{(2,0)}(M) \neq 0$ is equivalent to the familiar one $b_{2}^{+}(M)>1$.

${ }^{10}$ Remember that the 2-vector fields $\mathbf{X}$ and $\mathbf{Y}$ are not dynamical and they do not contribute to the Feynman integral to compute correlation functions. The analysis of zero modes is also unchanged and
} 
The theory on $\mathbb{R}^{4}$ in euclidean coordinates $\left(y^{1}, \ldots, y^{4}\right)$ with $z_{1}=y^{1}+i y^{2}$ and $z_{2}=y^{3}+i y^{4}$, suggests that the theory written in terms of $\mathcal{N}=1$ multiplets implies that the observables $Z$ are given by

$$
Z^{(2,0)}=\psi \psi+\bar{\omega} \bar{B} B, \quad Z^{(1,1)}=\lambda \psi+B F, \quad Z^{(0,2)}=\lambda \lambda
$$

where $\bar{\omega}$ is an anti-holomorphic 2-form. These observables are BRST-invariant with respect to the remnant supercharge $Q_{1}$ (after the supersymmetry breaking). This structure of observables comes from decomposition of a $\mathcal{N}=2$ vector multiplet in terms of $\mathcal{N}=1$ gauge multiplet $\left(A_{m}, \lambda\right)$ and a complex matter multiplet given by the scalar superfield $\Phi=(B, \psi)$. Here $A_{m}$ is a gauge field, $B$ is a complex scalar field and $\lambda$ and $\psi$ are spinor fields, all of them in the adjoint representation of the gauge group. The observables $Z^{(1,1)}$ and $Z^{(0,2)}$ come from the mentioned decomposition. However the presence of the term $\bar{\omega} \bar{B} B$ in $Z^{(2,0)}$ is a direct manifestation of the mass term that is added to the $\mathcal{N}=2$ Lagrangian in order to break supersymmetry. The introduction of a mass term in $\mathbb{R}^{4}$ reads

$$
\Delta L=-m \int d^{4} x d^{2} \theta \operatorname{Tr} \Phi^{2}-\text { h.c. }
$$

where the volume form is $d^{4} x d^{2} \theta=d^{2} z d^{2} \bar{z} d^{2} \theta$. This term preserves only $\mathcal{N}=1$ supersymmetry. It was proved in [9] that this perturbation $\Delta L$ to the Lagrangian is of the form: $\sum_{a} \alpha_{a} I\left(\Sigma_{a}\right)+\left\{Q_{1}, \cdot\right\}$. The canonical divisor $C \subset M$ is defined as the zero locus of $\omega$. Thus, in general on a curved Kähler manifold with $H^{(2,0)}(M) \neq 0$ the perturbed Lagrangian $\Delta L$ can be rewritten in terms of $\omega$ being a non-zero holomorphic form in $H^{(2,0)}(M)$ such that it vanishes on $C$. Consequently, the mass term vanishes precisely in the zeros of $\omega$ over the divisor (global cosmic strings) ${ }^{11}$.

Assume that $C=\bigcup_{y} C_{y}$, where $C_{y}$ is a Riemann surface for each $y$ such that $\omega$ has at most simple zeroes on $C_{y}$. One would estimate the contribution of the divisor (cosmic string) to the Donaldson-Witten invariants by considering the intersections $\Sigma \cap C_{y} \neq \emptyset$. In the intersection (which is assumed to be transverse) points $P$ one can insert operators $V_{y}(P)$. Thus, if $\#\left(\Sigma \cap C_{y}\right)$ is the intersection number of $\Sigma$ and $C_{y}$ is given by

$$
\#\left(\Sigma \cap C_{y}\right)=\int_{M} \theta_{\Sigma} \wedge \theta_{C_{y}}
$$

where $\theta_{\Sigma}$ is the Poincaré dual of $\Sigma$ and $\theta_{C_{y}}$ is the Poincaré dual of $C_{y}$. Then the operators $I(\Sigma)$ must be replaced by $\sum_{y} \#\left(\Sigma \cap C_{y}\right) V_{y}$. Here $V_{y}=V_{y}(P)$ is a local operator inserted in the intersection points $P$ 's between $\Sigma$ and $C_{y}$.

For the theory on the worldsheet (cosmic string) $C$ it is assumed that it has a mass gap and a chiral symmetry breaking with vacuum degeneracy determined by $\mathbb{Z}_{2}$. Fermionic zero modes on the divisor lead also to a non-vanishing anomaly inside the theory on $C_{y}$ which should cancel by other trapped fields along the string. Thus these

the dimension of the moduli space of instantons remains the same. The only change will be reflected in the definition of the observables.

${ }^{11}$ This cosmic string indeed captures chiral fermion zero modes of the field $\psi$ which propagates on the canonical divisor $C$. 
chiral fermions contributes to the path integral measure by a factor $t_{y}=(-1)^{d \varepsilon_{y}}$ where $\varepsilon_{y}=0,1$ and $d$ is the dimension of the gauge group. Gathering all together in Ref. [9] it was found that the Donaldson-Witten invariant is of the form

$$
\begin{gathered}
\left\langle\exp \left(\sum_{a} \alpha_{a} I(\Sigma)+\lambda \mathcal{O}\right)\right\rangle \\
=2^{\frac{1}{4}(7 \chi+11 \sigma)} \exp \left(\frac{1}{2} \sum_{a, b} \alpha_{a} \alpha_{b} \#\left(\Sigma_{a} \cap \Sigma_{b}\right)+2 \lambda\right) \cdot \prod_{y}\left(e^{\phi_{y}}+t_{y} e^{-\phi_{y}}\right) \\
+i^{\Delta} 2^{1+\frac{1}{4}(7 \chi+11 \sigma)} \exp \left(-\frac{1}{2} \sum_{a, b} \alpha_{a} \alpha_{b} \#\left(\Sigma_{a} \cap \Sigma_{b}\right)-2 \lambda\right) \cdot \prod_{y}\left(e^{-\phi_{y}}+t_{y} e^{\phi_{y}}\right),
\end{gathered}
$$

where

$$
\phi_{y}=\sum_{a} \alpha_{a} \#\left(\Sigma_{a} \cap C_{y}\right)
$$

and $\Delta=\frac{1}{2} d\left(\mathcal{M}_{D}\right)$.

The invariants (50) can be further generalized in the case the divisor components $C_{y}$ have singularities. Also the consideration of higher dimensional gauge groups leads to interesting generalizations. Both extensions were discussed in Ref. [9].

In summary, in the process of the obtention of (50) there were made a series of physical considerations. It was assumed cluster decomposition with a set of vacuum states, mass gap, chiral symmetry breaking, the smooth breaking of supersymmetry by the introduction of a mass term in the matter multiplet. All these assumptions are reasonable except the mass gap. It is well known that supersymmetric $\mathcal{N}=2$ YangMills theory don't have a mass gap. However this assumption make sense as one adds terms in the Lagrangian of the original $\mathcal{N}=2$ theory which leaves only one unbroken supersymmetry. The theory is $\mathcal{N}=1$, the mass gap is allowed and it gives precisely the necessary ingredient to interpret (50) as the Donaldson-Witten invariants of Kähler manifolds. This is the subject of the following subsection.

\subsection{Asymptotic Observables in Kähler Manifolds}

Before we proceed with the case of Kähler manifolds we make some considerations of general character about the asymptotic intersection of two asymptotic cycles. When a complex structure is defined in $M$, every form and vector field can be in general decomposed in a holomorphic, mixed and anti-holomorphic parts. Let $\left(z^{n}, z^{\bar{n}}\right)$ be complex coordinates on $M$ and $\left\{d z^{n}, d z^{\bar{n}}\right\}$ a basis for the cotangent space $T_{x}^{*} M$ and $\left\{\partial_{n}, \partial_{\bar{n}}\right\}$ a basis for the tangent space $T_{x} M$ at the point $x$. Then we can decompose our observable as [34]:

$$
Z=Z^{(0,2)}+Z^{(1,1)}+Z^{(2,0)},
$$

where in complex coordinates it looks like $Z^{(0,2)}=Z_{m n} d z^{m} \wedge d z^{n}, Z^{(1,1)}=Z_{m \bar{n}} d z^{n} \wedge d z^{\bar{n}}$ and $Z^{(2,0)}=Z_{\overline{m n}} d z^{\bar{m}} \wedge d z^{\bar{n}}$. In general every element corresponds to the decomposition of $\Omega^{p}(M)=\oplus_{p=r+s} \Omega^{(r, s)}(M)$, where $r$ and $s$ stands for the degrees of the corresponding 
holomorphic and anti-holomorphic components. One has a direct sum decomposition of $p$-vector fields $\mathcal{H}^{p}(M)=\oplus_{p=r+s} \mathcal{H}^{(r, s)}(M)$. Thus for $p=2$ :

$$
\mathbf{Y}=\mathbf{Y}^{(0,2)}+\mathbf{Y}^{(1,1)}+\mathbf{Y}^{(2,0)}
$$

where $\mathbf{Y}^{(0,2)}=Y^{m n} \partial_{m} \wedge \partial_{n}, \mathbf{Y}^{(1,1)}=Y^{m \bar{n}} \partial_{n} \wedge \partial_{\bar{n}}$ and $\mathbf{Y}^{(2,0)}=Y^{\overline{m n}} \partial_{\bar{m}} \wedge \partial_{\bar{n}}$. Now a 2-vector field can be constructed from vector fields as $\mathbf{Y}=Y_{1} \wedge Y_{2}$, where each $Y_{i}$ (for $i=1,2)$ is a vector field. Each $Y_{i}$ can be decomposed as the sum of a holomorphic and a anti-holomorphic part as follows, $Y_{i}=Y_{i}^{m} \partial_{m}+Y_{i}^{\bar{m}} \partial_{\bar{m}}$ then the 2-vector field $\mathbf{Y}$ takes the form

$$
\mathbf{Y}=Y_{1}^{m} Y_{2}^{n} \partial_{m} \wedge \partial_{n}+\left(Y_{1}^{m} Y_{2}^{\bar{n}}-Y_{1}^{\bar{n}} Y_{2}^{m}\right) \partial_{m} \wedge \partial_{\bar{n}}+Y_{1}^{\bar{m}} Y_{2}^{\bar{n}} \partial_{\bar{m}} \wedge \partial_{\bar{n}}
$$

Thus we can calculate the contraction of the 2-vector field $\mathbf{Y}$ and the observable $Z$. By the orthogonality relations between the basis, the asymptotic observable takes the following form

$$
\begin{aligned}
\widetilde{I}_{\mathbf{Y}}(\mu) & =\int_{M}\left[i_{\mathbf{Y}^{(0,2)}}\left(Z^{(0,2)}\right)+i_{\mathbf{Y}^{(1,1)}}\left(Z^{(1,1)}\right)+i_{\mathbf{Y}^{(2,0)}}\left(Z^{(2,0)}\right)\right] \mu \\
& =\int_{M}\left[Z_{m n} \cdot Y^{m n}+Z_{m \bar{n}} \cdot Y^{m \bar{n}}+Z_{\overline{m n}} \cdot Y^{\overline{m n}}\right] \mu .
\end{aligned}
$$

If the 2-vector fields are coming from the product of vector fields we have

$$
\widetilde{I}_{\mathbf{Y}}(\mu)=\int_{M}\left[Z_{m n} \cdot Y_{1}^{m} Y_{2}^{n}+Z_{m \bar{n}} \cdot\left(Y_{1}^{m} Y_{2}^{\bar{n}}-Y_{1}^{\bar{n}} Y_{2}^{m}\right)+Z_{\overline{m n}} \cdot Y_{1}^{\bar{m}} Y_{2}^{\bar{n}}\right] \mu .
$$

Moreover we can decompose the asymptotic observables into three different parts, one associated to a completely holomorphic part, one to a completely anti-holomorphic part and one to the mixed component.

For physical reasons [9] there are only three type of relevant observables. These are given by:

- The usual observable $\widetilde{I}(\Sigma)$ that contributes to the asymptotic intersection number (40) is given by: $\int_{M} i_{\mathbf{Y}^{(2,0)}}\left(Z^{(2,0)}\right) \mu+$ h.c., with $Z^{(2,0)}$ given in (47).

- The observable $I(\omega)=\int_{\Sigma} \omega$ arises from a non-vanishing mass term which breaks supersymmetry (from $\mathcal{N}=2$ to $\mathcal{N}=1$ ). The asymptotic version is written as $\int_{M} i_{\mathbf{Y}^{(2,0)}}\left(\omega^{(2,0)}\right) \mu+$ h.c. and it contributes to the asymptotic intersection number (49).

- Near intersection points of $\Sigma$ and $C_{y}$, where it is assumed to be inserted an operator $V(P)$ gives a term of the form $I(\theta)=\int_{M} \theta \wedge Z$. The natural form that couples to the asymptotic canonical divisor $\widetilde{C}_{y}$ is given by the first Chern class $J=c_{1}\left(\widetilde{C}_{y}\right)$ and it is a 2 -form of type $(1,1)$. It can be associated to a vector field $\mathbf{X}^{(1,1)}$. Thus we have that the observable is given by $\int_{M} i_{\mathbf{X}^{(1,1)}}(J) \mu$. 


\subsection{Invariants for Kähler Manifolds}

Let $\widetilde{C}$ be the disjoint union of a finite number of $\widetilde{C}_{y}$, where $\widetilde{C}_{y}$ is an asymptotic Riemann surface for each $y$ with simple zeroes. This asymptotic cycle can be defined in terms a free divergence 2 -vector field $\mathbf{X}$ and it is given by:

$$
\widetilde{C}_{y}(\mathbf{X})=\int_{M} i_{\mathbf{X}}(J) \mu
$$

where $J=c_{1}\left(C_{y}\right)$ and $\mathbf{X} \in \mathcal{H}^{(1,1)}$.

One can estimate the contribution of the divisor (cosmic string) to the DonaldsonWitten invariants of $M$ with flows. The contribution of the intersections $\widetilde{\Sigma}_{\mathbf{Y}} \cap \widetilde{C}_{y} \neq \emptyset$ can be computed as follows. The operators $\widetilde{I}_{\mathbf{Y}}(\mu)(x)$, inserted on the canonical divisor contribute precisely to the intersections of the cycles $\widetilde{\Sigma}$ with the canonical divisor $\widetilde{C}_{y}$ multiplied by a local operator $V(P)$. Thus it should make the replacement:

$$
\widetilde{I}_{\mathbf{Y}}(\mu)(x) \rightarrow \sum_{y} \#\left(\widetilde{\Sigma}_{\mathbf{Y}} \cap \widetilde{C}_{y}\right) V_{y}+\text { terms involving intersections of } \Sigma^{\prime} \mathrm{s} .
$$

The expectation values in the vacua of operators $V(P)$ are fixed by normalization. Thus the main contribution comes from $\#\left(\widetilde{\Sigma}_{\mathbf{Y}} \cap \widetilde{C}_{y}\right)$ which is given by the intersection number of two flows, one of them associated to the $\widetilde{\Sigma}$ and the other one to the asymptotic canonical divisor $\widetilde{C}_{y}$

$$
\#\left(\widetilde{\Sigma}_{\mathbf{Y}} \cap \widetilde{C}_{y}\right)=\int_{M_{1} \times M_{2}}\left(Z \wedge \Theta_{\mathbf{Y}}\right)\left(x_{1}\right) \wedge\left(J \wedge \Theta_{\mathbf{X}}\right)\left(x_{2}\right) \cdot \delta\left(x_{1}-x_{2}\right),
$$

where $\Theta_{\mathbf{Y}}=i_{\mathbf{Y}}(\mu)$ and $\Theta_{\mathbf{X}}=i_{\mathbf{X}}(\mu)$ are the Poincaré duals of $\widetilde{\Sigma}_{\mathbf{Y}}$ and $\widetilde{C}_{y}$ respectively. Here the measure $\mu$ is also invariant under the flow $\mathbf{X}$ generating the canonical divisor.

Gathering all together the previous considerations we have a form for the DonaldsonWitten invariants for Kähler manifolds with canonical divisor:

$$
\begin{gathered}
\left\langle\exp \left(\sum_{a} \alpha_{a} \widetilde{I}_{\mathbf{Y}}(\mu)+\lambda \mathcal{O}\right)\right\rangle \\
=2^{\frac{1}{4}(7 \chi+11 \sigma)} \exp \left(\frac{1}{2} \sum_{a, b} \alpha_{a} \alpha_{b} \#\left(\widetilde{\Sigma}_{\mathbf{Y}_{a}} \cap \widetilde{\Sigma}_{\mathbf{Y}_{b}}\right)+2 \lambda\right) \cdot \prod_{y}\left(e^{\widetilde{\phi}_{y}}+t_{y} e^{-\widetilde{\phi}_{y}}\right) \\
+i^{\Delta} 2^{1+\frac{1}{4}(7 \chi+11 \sigma)} \exp \left(-\frac{1}{2} \sum_{a, b} \alpha_{a} \alpha_{b} \#\left(\widetilde{\Sigma}_{\mathbf{Y}_{a}} \cap \widetilde{\Sigma}_{\mathbf{Y}_{b}}\right)-2 \lambda\right) \cdot \prod_{y}\left(e^{-\widetilde{\phi}_{y}}+t_{y} e^{\widetilde{\phi}_{y}}\right) .
\end{gathered}
$$

where $\widetilde{\phi}_{y}$ is given by Eq. (59) and $\Delta=\frac{1}{2} d\left(\mathcal{M}_{D}\right)$.

Finally, it is worth mentioning that one can generalize these expressions for more general Kähler manifolds with canonical divisors that don't have simple zeros. Further generalizations to non-simply connected manifolds, with $\pi_{1}(M) \neq 0$ and for higher dimensional gauge groups the reader can see, for instance [35]. 


\subsection{Examples}

1. Flows on hyper-Kähler manifolds with $\boldsymbol{H}^{(2,0)}(\boldsymbol{M}) \neq \mathbf{0}$. We start with $r$ divergence-free 2 -vector fields $Y_{a}$ with $a=1, \ldots, r$ on a 4 -torus $\mathbf{T}^{4}$. Then the asymptotic invariants associated to every 2-vector field can be computed through the correlation functions of a product of operators $\widetilde{I}\left(\mathbf{Y}_{a}\right)=\int_{M} i_{\mathbf{Y}_{a}}\left(Z^{(2,0)}\right) \mu_{a}+$ h.c $=\int_{M} i_{\mathbf{Y}_{a}}(\lambda \psi+\bar{\omega} \bar{B} B) \mu_{a}+$ h.c.. Thus to this configurations of flows we get the following invariant by using the Hodge structure of the torus: $h^{1,0}=2, h^{2,0}=1$. The invariant is given by

$$
\begin{aligned}
\left\langle\exp \left(\sum_{a} \alpha_{a} \tilde{I}\left(Y_{a}\right)+\lambda \mathcal{O}\right)\right\rangle & =\exp \left(\frac{1}{2} \sum_{a, b} \alpha_{a} \alpha_{b} \#\left(\widetilde{\Sigma}_{\mathbf{Y}_{a}} \cap \widetilde{\Sigma}_{\mathbf{Y}_{b}}\right)+2 \lambda\right) \\
& +\exp \left(-\frac{1}{2} \sum_{a, b} \alpha_{a} \alpha_{b} \#\left(\widetilde{\Sigma}_{\mathbf{Y}_{a}} \cap \widetilde{\Sigma}_{\mathbf{Y}_{b}}\right)-2 \lambda\right)
\end{aligned}
$$

where \#( $\left.\widetilde{\Sigma}_{\mathbf{Y}_{a}} \cap \widetilde{\Sigma}_{\mathbf{Y}_{b}}\right)$ is the asymptotic intersection number. Thus we find that $\#\left(\widetilde{\Sigma}_{\mathbf{Y}_{a}} \cap \widetilde{\Sigma}_{\mathbf{Y}_{b}}\right)$ is given by Eq. (44). In the specific case of $\mathbf{T}^{4}$ we have 2 cycles of dimension two, one holomorphic and the other anti-holomorphic. The vector fields $\mathbf{Y}_{a}$ are wrapped on these homology cycles. There is one-dimensional homology cycles and one can introduce vector fields whose orbits coincide with these cycles. One can construct asymptotic invariants associated with them and compute their contribution to the correlation functions. But we are not interested in this addition in the present paper. However it is interesting to remark that 2-vector fields can be constructed from the wedge product of two of these vector fields and we have constructed observables by using (56). Thus we find that our invariant can be computed by using Eq. (40).

For the case of $\mathrm{K} 3$, where $h^{1,0}=0, h^{2,0}=1$, we don't have nonsingular vector fields (since the Euler characteristic is 24). However we have only intrinsic 2vector fields. Thus there will be not 2 -vector fields constructed from 1-vector fields as in the previous example. In this case the invariant is given by

$$
\begin{aligned}
\left\langle\exp \left(\sum_{a} \alpha_{a} \tilde{I}\left(Y_{a}\right)+\lambda \mathcal{O}\right)\right\rangle & =C\left[\exp \left(\frac{1}{2} \sum_{a, b} \alpha_{a} \alpha_{b} \#\left(\widetilde{\Sigma}_{\mathbf{Y}_{a}} \cap \widetilde{\Sigma}_{\mathbf{Y}_{b}}\right)+2 \lambda\right)\right. \\
& \left.-\exp \left(-\frac{1}{2} \sum_{a, b} \alpha_{a} \alpha_{b} \#\left(\widetilde{\Sigma}_{\mathbf{Y}_{a}} \cap \widetilde{\Sigma}_{\mathbf{Y}_{b}}\right)-2 \lambda\right)\right]
\end{aligned}
$$

where $C=1 / 4$. The asymptotic intersection number is also given only by Eq. (44) and the sum involves also normal and asymptotic self-intersection numbers.

2. Hilbert Modular surfaces [36]. Let $\mathbb{H} \subset \mathbb{C}$ denote the upper half-plane with the Poincaré metric. Let $K:=\mathbb{Q}(\sqrt{2})$ be the totally real quadratic number field obtained by adding $\sqrt{2}$ to $\mathbb{Q}$. The ring of integers $\mathbb{Z}(\sqrt{2})$ is the ring of real numbers of the form $m+n \sqrt{2}, m, n \in \mathbb{Z}$. Let $\sigma$ be the nontrivial Galois 
automorphism of $K$ given explicitly by $\sigma(a+b \sqrt{2})=a-b \sqrt{2}, a, b \in \mathbb{Q}$. Let us consider the group $\Gamma:=\operatorname{PSL}(2, \mathbb{Z}(\sqrt{2}))$. Let $\bar{\sigma}: \Gamma \rightarrow \Gamma$ be the induced automorphism on $\Gamma$.

$\Gamma$ acts properly and discontinuously on $\mathbb{H} \times \mathbb{H}$ as follows: $\gamma(z, w)=(\gamma(z), \sigma(\gamma)(w))$. The quotient is an orbifold of dimension four which is not compact but it has finite volume (with respect to the induced metric coming from the product metric on $\mathbb{H} \times \mathbb{H}$ ). The ends (cusps) of Hilbert modular surfaces of real quadratic number fields are manifolds which are of form $M^{3} \times[0, \infty)$ where $M^{3}$ is a compact solvable 3 -manifold which fibers over the circle with fibre a torus $\mathbb{T}^{2}$. The number of such ends is equal to the class number of the field [36]. For $\mathbb{Q}(\sqrt{2})$ there is only one cusp and $M^{3}$ is the mapping torus of the automorphism of the 2-torus induced by the matrix $A=\left(\begin{array}{ll}2 & 1 \\ 1 & 1\end{array}\right)$. Thus $M^{3}$ fibers over the circle. In fact, the sugbroup $\Lambda \subset \Gamma$ consisting of affine transformations of the form $z \mapsto(1+\sqrt{2})^{r} z+m+n \sqrt{2}$, $r, m, n \in \mathbb{Z}$ is the semidirect product $\mathbb{Z} \ltimes_{A}(\mathbb{Z} \times \mathbb{Z})$ which is the solvable fundamental group of $M^{3}$. The universal cover of $M^{3}$ is a solvable simply connected 3-dimensional Lie group whose Lie algebra is generated by three left-invariant vector fields $\mathbf{X}, \mathbf{Y}$ and $\mathbf{Z}$ whose Lie brackets satisfy $[\mathbf{X}, \mathbf{Y}]=a \mathbf{Y},[\mathbf{X}, \mathbf{Z}]=-a \mathbf{Z}$ and $[\mathbf{Y}, \mathbf{Z}]=0$, for some constant $a>0$. The commuting vector fields $\mathbf{Y}$ and $\mathbf{Z}$ descend to vector fields in $M^{3}$ which are tangent to the torus fibers of the fibration of $M^{3}$ over the circle. The flow generated by $\mathbf{X}$ is an Anosov flow. The vector fields $\mathbf{Y}$ and $\mathbf{Z}$ generate two flows tangent to 1-dimensional foliations $L_{1}$ and $L_{2}$. These flows are homologous to zero and the Arnold's self-linking number of both is zero.

By a theorem of Selberg [37] $\Gamma$ contains a finite index subgroup $\tilde{\Gamma}$ which acts freely on $\mathbb{H} \times \mathbb{H}$. The quotient manifold $M^{4}(\tilde{\Gamma}):=\tilde{\Gamma} / \mathbb{H} \times \mathbb{H}$ is a non compact manifold of finite volume with a finite number of cusps depending upon the Selberg subgroup. The action of $P S L(2, \mathbb{Z}(\sqrt{2}))$ preserves the natural foliations of $\mathbb{H} \times \mathbb{H}$ whose leaves are, respectively, of the form $\mathbb{H} \times\{w\}$ and $\{z\} \times \mathbb{H}$. These foliations descend to $M^{4}(\tilde{\Gamma})$ to a pair of 2-dimensional foliations $\mathcal{F}_{\text {horizontal }}$ and $\mathcal{F}_{\text {vertical }}$ which are mutually transverse and each has dense leaves. Furthermore, since the action of $\Gamma$ is by isometries, the foliations $\mathcal{F}_{\text {horizontal }}$ and $\mathcal{F}_{\text {vertical }}$ are transversally Riemannian and thus both have natural transverse measures.

Now we can do two things to obtain examples of 4-manifolds with (possibly singular) foliations.

- We can compactify $M^{4}(\tilde{\Gamma})$ à la Hirzebruch [38] by adding one point at infinity for each cusp. The resulting space is an algebraic surface with singularities at the cusps and the link of each singularity is the corresponding solvmanifold. After desingularizing one obtains a smooth algebraic surface which is therefore a Kähler surface. The foliations $\mathcal{F}_{\text {horizontal }}$ and $\mathcal{F}_{\text {vertical }}$ lift to the desingularized manifold to foliations with singularities at the cusps. There are important relations of these constructions with $K 3$ surfaces [39].

- One can "cut" the manifold at each cusp, to obtain a compact manifold with 
boundary and each component of the boundary is a solvmanifold described before. In other words, we remove a conic open neighborhood of each cusp whose boundary is the corresponding solvmanifold at the cusp. Now we can take the double to obtain a compact closed manifold with a pair of transversally Riemannian foliations with dense leaves (since in the double the foliations can be glued differentiably). Both foliations meet transversally the solvmanifolds and determine two flows in them. For the case $K=$ $\mathbb{Q}(\sqrt{2})$ we obtain a compact 4-manifold with two transversally Riemannian foliations which meet the solvmanifold in the foliations $L_{1}$ and $L_{2}$ above. Therefore: each of the foliations $\mathcal{F}_{\text {horizontal }}$ and $\mathcal{F}_{\text {vertical }}$ has self-intersection zero (since $L_{1}$ and $L_{2}$ have self intersection zero).

Of course one can construct examples as above using any totally real quadratic field and the group $\operatorname{PSL}\left(2, \mathfrak{O}_{K}\right)$, where $\mathfrak{O}_{K}$ is the ring of integers of $K$.

3. Elliptic K3 surfaces end elliptic surfaces. Let $S$ be an elliptic surface with Kodaira fibration $\pi: S \rightarrow \Sigma_{g}$, where $\Sigma_{g}$ is an algebraic curve of genus $g$. The fibres are elliptic curves except for a finite number of singular fibres which are rational curves. The fibration provides us with a singular foliation as mentioned in the remark above. There is a canonical choice for a transverse measure $\mu$ which is obtained from the Poincaré metric via the uniformization theorem applied to $\Sigma_{g}$ : if $\tau$ is a 2-disk which is transversal to the regular part of the foliation its measure is the hyperbolic area of $\pi(\tau)$. Then we can apply our results to the triple $(S, \mathcal{F}, \mu)$. One modification of elliptic surfaces can be obtained by the so-called logarithmic transformation. Using logarithmic transformation one can change the Kodaira dimension and turn an algebraic surface into a non algebraic surface.

Particular cases of elliptic surfaces are the $K 3$ surfaces, Enriques surfaces and the Dolgachev surfaces. We recall that Dolgachev $X_{p}$ surfaces depend on an integer $p$ were used by Donaldson to obtain the first examples $X_{2}$ and $X_{3}$ of manifolds which are homeomorphic but not diffeomorphic [39], [4]. From the above two questions arise:

- How do our invariants change after performing a logarithmic transformation on an elliptic surface?

- Can we detect exotic differentiable structures by our invariants?

4. Symplectic 4-manifolds and Lefschetz fibrations and pencils. By a result of Donaldson [40] every symplectic 4-manifold admits a Lefschetz fibration and these fibrations are an essential tool for the study of symplectic 4-manifolds. As the previous example, one has a triple $\left(M^{4}, \mathcal{F}, \mu\right)$, where $\mathcal{F}$ is the (possibly singular) foliation determined by the Lefschetz fibration and $\mu$ is a transverse measure coming from a choice of an area form from a Riemannian metric on the base surface. The question is how to compute our invariants and how can they be used to study symplectic manifolds. 


\section{Survey on Seiberg-Witten Invariants}

Another example of the theories which can be constructed through the Mathai-Quillen formalism is the cohomological field theory describing Seiberg-Witten monopoles [11]. The geometric data consists of the square root of the (determinant) line bundle $L^{1 / 2}$ over a four-manifold $M$ with an abelian gauge connection $A$ with curvature $F_{A}=d A$. We have also the tensor product $S^{ \pm} \otimes L^{1 / 2}$ of $L^{1 / 2}$ with the spin bundle $S^{ \pm}$, which exist whenever $M$ is a spin manifold i.e. $w_{2}(M)=0$ (for more details on the spin structure see, $[41,42])$. This tensor product is even well defined if $M$ is not a spin manifold. In addition we have a section $\psi_{\alpha} \in \Gamma\left(S^{+} \otimes L^{1 / 2}\right)$. The Seiberg-Witten equations are

$$
F_{\alpha \beta}^{+}=-\frac{i}{2} \bar{\psi}_{(\alpha} \psi_{\beta)}, \quad D_{\alpha \dot{\alpha}} \psi^{\alpha}=0,
$$

where $F^{+}$is the self-dual part of the curvature $F_{A}$. Here $\alpha, \beta$ are spinorial indices instead of vector ones $\mu$ and they are related by $A_{\mu}=\sigma_{\mu}^{\alpha \dot{\alpha}} A_{\alpha \dot{\alpha}}$.

The moduli space $\mathcal{M}_{S W}$ of solutions to the Seiberg-Witten equations will be denoted as $\mathcal{M}_{S W} \subset \mathcal{A} \times \Gamma\left(S^{+} \otimes L^{1 / 2}\right) / \mathcal{G}$, where $\mathcal{A}$ is the space of abelian connections on $L^{1 / 2}$ and $\mathcal{G}$ is the gauge group of the $U(1)$-bundle, i.e. $\mathcal{G}=\operatorname{Map}(M, U(1))$. This moduli problem can be described in terms of the Mathai-Quillen construction. In this case the vector bundle is also trivial $\mathcal{V}=\mathcal{M} \times \mathcal{F}$, where $\mathcal{F}$ is the fibre. For the monopole case $\mathcal{F}=\Lambda^{2,+}(M) \otimes \Gamma\left(S^{-} \otimes L^{1 / 2}\right)$. The section $s$ is given by

$$
s(A, \psi)=\left(\frac{1}{\sqrt{2}}\left(F_{\alpha \beta}^{+}+\frac{i}{2} \bar{\psi}_{(\alpha} \psi_{\beta)}\right), D_{\alpha \dot{\alpha}} \psi^{\alpha}\right),
$$

where $D_{\alpha \dot{\alpha}} \psi_{\beta}=\sigma_{\alpha \dot{\alpha}}^{\mu}\left(\partial_{\mu}+i A_{\mu}\right) \psi_{\beta}$. The zero section determines precisely the SeibergWitten equations.

The dimension $d\left(\mathcal{M}_{S W}\right)$ of the moduli space $\mathcal{M}_{S W}$ can be obtained from an index theorem

$$
d\left(\mathcal{M}_{S W}\right)=\lambda^{2}-\frac{2 \chi+3 \sigma}{4},
$$

where $\lambda=\frac{1}{2} c_{1}(L)$ (being $c_{1}(L)$ the first Chern class), $\chi$ and $\sigma$ are the Euler characteristic and the signature of $M$ respectively. The Mathai-Quillen construction [31] provides with a set of fields $A_{\mu}, \psi_{\mu}, \phi, \chi_{\mu \nu}, H_{\mu \nu}, \eta, \psi_{\alpha}, \mu_{\alpha}, v_{\dot{\alpha}}$ and $h_{\dot{\alpha}}$ of different ghost number. This set of fields will be denoted for short as $\mathcal{X}$. The Lagrangian can be read off from the exponential of the Thom class and is given by $[12,43]$

$$
\begin{gathered}
L_{S W}=\int_{M} e\left(g^{\mu \nu} D_{\mu} \bar{\psi}^{\alpha} D_{\nu} \psi_{\alpha}+\frac{1}{4} R \bar{\psi}^{\alpha} \psi_{\alpha}+\frac{1}{2} F^{+\alpha \beta} F_{\alpha \beta}^{+}-\frac{1}{8} \bar{\psi}^{(\alpha} \psi^{\beta)} \bar{\psi}_{(\alpha} \psi_{\beta)}\right) \\
+i \int_{M}\left(\lambda \wedge * d^{*} d \phi-\frac{1}{\sqrt{2}} \chi \wedge * \rho^{+} d \psi\right)+\int_{M}\left[i \phi \lambda \bar{\psi}^{\alpha} \psi_{\alpha}+\frac{1}{2 \sqrt{2}} \chi^{\alpha \beta}\left(\bar{\psi}_{(\alpha} \mu_{\beta)}+\bar{\mu}_{(\alpha} \psi_{\beta)}\right)\right. \\
\left.\left.-\frac{i}{2}\left(v^{\dot{\alpha}} D_{\alpha \dot{\alpha}} \mu^{\alpha}-\mu^{\alpha} D_{\alpha \dot{\alpha}} v^{\dot{\alpha}}\right)-\frac{i}{2}\left[\bar{\psi}^{\alpha} \psi_{\alpha \dot{\alpha}} v^{\dot{\alpha}}\right]+\frac{1}{2} \eta\left(\bar{\mu}^{\alpha} \psi_{\alpha}\right)-\bar{\psi}^{\alpha} \mu_{\alpha}\right)+\frac{i}{4} \phi \bar{v}^{\dot{\alpha}} v_{\dot{\alpha}}-\lambda \bar{\mu}^{\alpha} \mu_{\alpha}\right] .
\end{gathered}
$$

The observables are products of BRST invariant operators which are cohomologically non-trivial

$$
d \Theta_{p}^{n}=\left\{\mathcal{Q}, \Theta_{p+1}^{n}\right\} .
$$


The $\mathcal{Q}$-invariant operators are [43]

$$
\begin{gathered}
\mathcal{O}_{n}^{\gamma_{0}}=\Theta_{0}^{n}(x) \\
\mathcal{O}_{n}^{\gamma_{1}}=\int_{\gamma_{1}} \Theta_{1}^{n}, \quad \mathcal{O}_{n}^{\gamma_{2}}=\int_{\gamma_{2}} \Theta_{2}^{n} \\
\mathcal{O}_{n}^{\gamma_{3}}=\int_{\gamma_{3}} \Theta_{3}^{n}, \quad \mathcal{O}_{n}^{\gamma_{4}}=\int_{M} \Theta_{4}^{n},
\end{gathered}
$$

where

$$
\begin{gathered}
\Theta_{0}^{n}=\left(\begin{array}{c}
n \\
0
\end{array}\right) \phi^{n}, \quad \Theta_{1}^{n}=\left(\begin{array}{c}
n \\
1
\end{array}\right) \phi^{n-1} \psi, \\
\Theta_{2}^{n}=\left(\begin{array}{c}
n \\
2
\end{array}\right) \phi^{n-2} \psi \wedge \psi+\left(\begin{array}{c}
n \\
1
\end{array}\right) \phi^{n-1} \psi \wedge F \\
\Theta_{3}^{n}=\left(\begin{array}{c}
n \\
3
\end{array}\right) \phi^{n-3} \psi \wedge \psi \wedge \psi+2\left(\begin{array}{c}
n \\
2
\end{array}\right) \phi^{n-2} \psi \wedge F \\
\Theta_{4}^{n}=\left(\begin{array}{c}
n \\
4
\end{array}\right) \phi^{n-4} \psi \wedge \psi \wedge \psi \wedge \psi+3\left(\begin{array}{c}
n \\
3
\end{array}\right) \phi^{n-3} \psi \wedge \psi \wedge F+\left(\begin{array}{c}
n \\
2
\end{array}\right) \phi^{n-2} F \wedge F .
\end{gathered}
$$

Here $\Theta_{0}^{n}(x)$ is constructed with a gauge and $\mathcal{Q}$ invariant field $\phi$. All other observables are descendants obtained from it [43]. As in the Donaldson-Witten case the construction establishes an isomorphism between the BRST cohomology $H_{B R S T}^{*}(\mathcal{Q})$ and the de Rham cohomology $H_{d R}^{*}(M)$. To be more precise the analogue to the Donaldson map is: $\delta_{S W}: H_{p}(M) \rightarrow H^{2-p}\left(\mathcal{M}_{S W}\right)$ given in terms of the first Chern class of $\mathcal{V}$. The observables (68) are BRST invariant (BRST closed) and the BRST commutator only depends of the homology class. This can be shown by following a similar procedure as we did in the Donaldson case (see Eqs. (10) and (11)).

The correlation functions of $r$ operators are written as

$$
\begin{aligned}
\left\langle\mathcal{O}_{n}^{\gamma_{p_{1}}} \cdots \mathcal{O}_{n}^{\gamma_{p_{r}}}\right\rangle & =\left\langle\prod_{j=1}^{r} \int_{\gamma_{p_{j}}} \Theta_{p_{j}}^{n}\right\rangle \\
& =\int \mathcal{D} \mathcal{X} \exp \left(-L_{S W} / e^{2}\right) \prod_{j=1}^{r} \int_{\gamma_{p_{j}}} \Theta_{p_{j}}^{n} .
\end{aligned}
$$

These are the Seiberg-Witten invariants in the path integral representation [12, 43]. They are topological invariants and also invariants of the smooth structure of $M$. After integration over the non-zero modes one has:

$$
\begin{aligned}
\left\langle\mathcal{O}_{1}^{\gamma_{p_{1}}} \ldots \mathcal{O}_{r}^{\gamma_{p_{r}}}\right\rangle & =\left\langle\prod_{j=1}^{r} \int_{\gamma_{p_{j}}} \Theta_{p_{j}}^{n}\right\rangle \\
& =\int_{\mathcal{M}_{S W}} \nu_{p_{1}} \wedge \cdots \wedge \nu_{p_{r}}
\end{aligned}
$$


where $\nu_{p_{j}}=\delta_{S W}\left(\gamma_{p_{j}}\right)$. The possible values of $p_{j}$ are $0,1,2$. Thus, for $p_{j}=1,2$, we can rewrite the previous equation as

$$
\left\langle\mathcal{O}_{1}^{\gamma_{1}} \cdots \mathcal{O}_{r}^{\gamma_{1}} \cdot \mathcal{O}_{1}^{\gamma_{2}} \cdots \mathcal{O}_{d / 2}^{\gamma_{2}}\right\rangle=\int_{\mathcal{M}_{S W}} \nu_{1_{1}} \wedge \cdots \wedge \nu_{1_{r}} \wedge \phi_{\Sigma}^{d / 2}
$$

where $\phi_{\Sigma}$ are 2-forms on the SW moduli space. For simply connected manifolds $\pi_{1}(M)=0$ the relevant cycles are of dimension $p=2$. The Seiberg-Witten invariants can be also written in terms of differential forms in the moduli space $\mathcal{M}_{S W}$ in the form $[12,43]$

$$
\left\langle\mathcal{O}_{1}^{\gamma_{2}} \cdots \mathcal{O}_{d / 2}^{\gamma_{2}}\right\rangle=\int_{\mathcal{M}_{S W}} \phi_{\Sigma}^{d / 2}
$$

\section{Seiberg-Witten Invariants for Flows}

In order to incorporate flows in the Seiberg-Witten theory we define

$$
\widetilde{\mathcal{O}}_{\mathbf{Y}_{p}}(\mu)=\int_{M} i_{\mathbf{Y}_{p}}\left(\Theta_{p}^{n}\right) \mu_{p}(x)
$$

where $\mu_{p}$ is the invariant volume form. We can interpret the integral as the averaged asymptotic cycles on $M$ by the Schwartzman theorem [18], $i_{\mathbf{Y}_{p}}(\mu)$ is a closed $(4-p)$ form, from which we will obtain a asymptotic $p$-cycle $\widetilde{\gamma}_{p}$ by Poincaré duality (an element of the $\left.H_{p}(M, \mathbb{R})\right)$.

Let $\mathbf{Y}_{p}$ be $p$-vector fields with $p=0,1,2$, then the expression (74) defines the asymptotic observables as

$$
\widetilde{\mathcal{O}}_{\mathbf{Y}_{1}}^{n}\left(\mu_{1}\right)=\int_{M} i_{\mathbf{Y}_{1}}\left(\Theta_{1}^{n}\right) \mu_{1}, \quad \widetilde{\mathcal{O}}_{\mathbf{Y}_{2}}^{n}\left(\mu_{2}\right)=\int_{M} i_{\mathbf{Y}_{2}}\left(\Theta_{2}^{n}\right) \mu_{2}
$$

where $\Theta_{p}^{n}$ are given by (69).

Follow the procedure we did in Eq. (25), it is an easy matter to check that these asymptotic observables $\widetilde{\mathcal{O}}_{\mathbf{Y}_{p}}\left(\mu_{p}\right)$ are $\mathcal{Q}$-invariant (BRST).

For an oriented manifold $M$ with $p_{j}$-vectors fields $\mathbf{Y}_{p_{j}}\left(p_{j}=0,1,2\right)$, with $\sum_{j=1}^{r} p_{j}=$ $\mathrm{d}\left(\mathcal{M}_{\mathrm{SW}}\right)$ and probability invariant measure, the $r$-point correlation functions for the flow generated $\mathbf{Y}_{p_{j}}$ and $\mu_{p_{j}}$ are given by

$$
\left\langle\widetilde{\mathcal{O}}_{\mathbf{Y}_{p_{1}}}^{n}\left(\mu_{p_{1}}\right) \cdots \widetilde{\mathcal{O}}_{\mathbf{Y}_{p_{r}}}^{n}\left(\mu_{p_{r}}\right)\right\rangle=\int(\mathcal{D} \mathcal{X}) \exp \left(-L_{S W} / e^{2}\right) \prod_{j=1}^{r} \int_{M} i_{\mathbf{Y}_{p_{j}}}\left(\Theta_{p_{j}}^{n}\right) \mu_{p_{j}} .
$$

This expression is reduced to the ordinary Seiberg-Witten invariants (71), when the measure is supported on the cycles. This means if $\mu_{p}=\sum_{j=1}^{r} \mu_{p_{j}}$ where each $\mu_{p}$ is distributed uniformly over the cycles of $M$. 
Similarly to the Donaldson case let us assume that the only zero modes correspond to the abelian gauge field $A_{\mu}$ and its BRST-like companion $\psi_{\mu}$. Following a similar procedure as in the Donaldson case to compute the partition function we get

$$
\begin{aligned}
\left\langle\widetilde{\mathcal{O}}_{\mathbf{Y}_{p_{j}}}\left(\mu_{p_{j}}\right)\right\rangle & =\int_{\mathcal{M}_{\mathcal{S W}}} d a_{1} \ldots d a_{n} d \psi^{1} \ldots d \psi^{n} \widetilde{\Phi}_{i_{1} \ldots i_{n}}\left(a_{i}, \mathbf{Y}_{p_{j}}\right) \psi^{i_{1}} \ldots \psi^{i_{n}} \\
& =\int_{\mathcal{M}_{\mathcal{S W}}} \widetilde{\Phi}_{\mathbf{Y}_{d(\mathcal{M})}},
\end{aligned}
$$

where $\widetilde{\Phi}_{\mathbf{Y}_{p_{j}}}\left(\mu_{p_{j}}\right)=\widetilde{\Phi}_{i_{1} \cdots i_{n}}\left(a_{i}, \mathbf{Y}_{p_{j}}\right) \psi^{i_{1}} \ldots \psi^{i_{n}}, a$ 's are the zero modes of the gauge field and $\psi$ 's are the zero modes of the fermionic field and $\widetilde{\Phi}\left(a, \mathbf{Y}_{p_{j}}\right)$ is a function that only depends of the zero modes of the gauge field and contains the information of the flow.

We integrate out $a_{i}$ 's and obtain a $n$-form $\widetilde{\Phi}$ defined in the moduli space. Now suppose that $\widetilde{\mathcal{O}}=\widetilde{\mathcal{O}}_{\mathbf{Y}_{p_{1}}}\left(\mu_{p_{1}}\right) \cdots \widetilde{\mathcal{O}}_{\mathbf{Y}_{p_{r}}}\left(\mu_{p_{r}}\right)$ with $\sum_{p, j} p_{j}=n=d\left(\mathcal{M}_{S W}\right)$ and $p_{j}$ is the number of zero modes of $\widetilde{\mathcal{O}}_{\mathbf{Y}_{p_{j}}}\left(\mu_{p_{j}}\right)$. These functions define forms in the moduli space in the following way

$$
\begin{aligned}
\left\langle\widetilde{\mathcal{O}}_{\mathbf{Y}_{p_{1}}}\left(\mu_{p_{1}}\right) \cdots \widetilde{\mathcal{O}}_{\mathbf{Y}_{p_{r}}}\left(\mu_{p_{r}}\right)\right\rangle & =\int_{\mathcal{M}_{S W}} \widetilde{\Phi}_{\mathbf{Y}_{p_{1}}} \wedge \cdots \wedge \widetilde{\Phi}_{\mathbf{Y}_{p_{r}}} \\
& =\int_{\mathcal{M}_{S W}} \widetilde{\nu}_{\mathbf{Y}_{p_{1}}} \wedge \cdots \wedge \widetilde{\nu}_{\mathbf{Y}_{p_{r}}} .
\end{aligned}
$$

In the simply connected case $\left(\pi_{1}(M)=0\right)$, the important observables are those associated with cycles of zero dimension $\gamma_{0}$ and of dimension two $\gamma_{2}$. In general a $k_{\gamma}$-cycle has associated an operator (form) with ghost number $U=2-k_{\gamma}$, this is the analog of the Donaldson map $H_{k}(M) \rightarrow H^{2-k}\left(\mathcal{M}_{S W}\right)$. Finally it is easy to see that for $k=2$ the product of $r$ operators yields

$$
\begin{aligned}
\left\langle\widetilde{\mathcal{O}}_{\mathbf{Y}_{1}}\left(\mu_{1}\right) \cdots \widetilde{\mathcal{O}}_{\mathbf{Y}_{d / 2}}\left(\mu_{d / 2}\right)\right\rangle & =\int_{\mathcal{M}_{S W}} \widetilde{\nu}_{\mathbf{Y}_{1}} \wedge \cdots \wedge \widetilde{\nu}_{\mathbf{Y}_{d / 2}} \\
& =\#\left(H_{\widetilde{\Sigma}_{\mathbf{Y}_{1}}} \cap \ldots \cap H_{\widetilde{\Sigma}_{\mathbf{Y}_{d / 2}}}\right),
\end{aligned}
$$

where we have assumed the notation $\mathbf{Y}_{2_{j}}=\mathbf{Y}_{j}$ and $\mu_{2_{j}}=\mu_{j}$ for asymptotic 2-cycles.

These ideas can be generalized by considering a twisted version of the Yang-Mills theory with non-abelian gauge group and this leads to non-abelian monopole equations $[44,45]$. We consider the general case, when $M$ is not Spin but a $\operatorname{Spin}_{c} \operatorname{manifold}_{\text {. For }}$ one hypermultiplet the equations for a non-abelian connection $A_{\mu}$ coupled to a spinor $M_{\alpha} \in \Gamma\left(S^{+} \otimes L^{1 / 2} \otimes E\right)$, where $S^{+}$is the spin bundle, $L^{1 / 2}$ is the determinant line bundle of the $\operatorname{Spin}_{c}$ structure and $E$ is the vector bundle associated to a principal $G$ bundle via some representation of the gauge group. Then the equations for the moduli space are given by

$$
F_{\dot{\alpha} \dot{\beta}}^{+a}+4 i \bar{M}_{(\dot{\alpha}}\left(T^{a}\right) M_{\dot{\beta})}=0, \quad\left(\nabla_{E}^{\alpha \dot{\alpha}} \bar{M}_{\dot{\alpha}}\right)=0,
$$

$T^{a}$ are the generators of the Lie algebra, $\nabla_{E}^{\alpha \dot{\alpha}}$ is the Dirac operator constructed with the covariant derivative with respect to the gauge connection $A_{\mu}$. Thus it is possible to 
extend the set of observables (75) for the non-abelian case. Similar computations can be done for obtaining the Seiberg-Witten invariants associated to non-abelian monopoles [44] for the case of compact Kähler manifolds following Ref. [45] and making a similar procedure as described in Sec. 5.2.

\subsection{Relation to Donaldson Invariants}

The computation of Donaldson-Witten and Seiberg-Witten invariants for Kähler manifolds can be obtained by physical methods. The difference lies in the underlying physics of the dynamics of $\mathcal{N}=2$ supersymmetric gauge theories. For the Donaldson-Witten case it was needed to add a mass term to soft breaking supersymmetry. This introduces a non-trivial canonical divisor defined as the zero locus of the mass term. However for the Seiberg-Witten case this is not necessary. The underlying dynamics describing the strong coupling limit of the gauge theory was elucidated in [10] through the implementation of S-duality. The dual theory was used later by Witten in Ref. [11] to find new invariants of four manifolds, the Seiberg-Witten invariants. In that paper it was found a relation between both invariants. This subsection contains the description of this relation when there are non-singular global flows on the manifold.

The relevant ingredients are the operators $\widetilde{I}_{\mathbf{Y}_{a}}$ and $\mathcal{O}$ inserted in $M$. Then the Donaldson invariants take the following form

$$
\begin{gathered}
\left\langle\exp \left(\sum_{a} \alpha_{a} \widetilde{I}_{\mathbf{Y}_{a}}\left(\mu_{a}\right)+\lambda \mathcal{O}\right)\right\rangle \\
=2^{1+\frac{1}{4}(7 \chi+11 \sigma)}\left[\exp \left(\frac{1}{2} \widetilde{v}^{2}+2 \lambda\right) \sum_{\widetilde{x}} \widetilde{S W}(\widetilde{x}) e^{\widetilde{v} \cdot \widetilde{x}}+i^{\Delta} \exp \left(-\frac{1}{2} \widetilde{v}^{2}-2 \lambda\right) \cdot \sum_{\widetilde{x}} \widetilde{S W}(\widetilde{x}) e^{-i \widetilde{v} \cdot \widetilde{x}}\right]
\end{gathered}
$$

where $\alpha_{a}$ and $\lambda$ are complex numbers,

$$
\begin{gathered}
\widetilde{v}^{2}=\sum_{a, b} \alpha_{a} \alpha_{b} \#\left(\widetilde{\Sigma}_{\mathbf{Y}_{a}} \cap \widetilde{\Sigma}_{\mathbf{Y}_{b}}\right) \\
\widetilde{v} \cdot \widetilde{x}=\sum_{a} \alpha_{a} \#\left(\widetilde{\Sigma}_{\mathbf{Y}_{a}} \cap \widetilde{x}\right)
\end{gathered}
$$

and $\widetilde{S W}$ is the asymptotic version of the Seiberg-Witten invariant (79).

Here $\#\left(\widetilde{\Sigma}_{\mathbf{Y}_{a}} \cap \widetilde{x}\right)$ is the asymptotic intersection number between $\widetilde{\Sigma}_{\mathbf{Y}_{a}}$ and a $\widetilde{x}$ and it is given by

$$
\#\left(\widetilde{\Sigma}_{\mathbf{Y}_{a}} \cap \widetilde{x}\right)=\int_{M_{1} \times M_{2}}\left(\Theta_{2} \wedge \eta_{\mathbf{Y}_{a}}\right)\left(x_{1}\right) \wedge\left(x \wedge \eta_{\mathbf{X}}\right)\left(x_{2}\right) \cdot \delta\left(x_{1}-x_{2}\right),
$$

where $\eta_{\mathbf{Y}_{a}}$ and $\eta_{\mathbf{X}}$ are the Poicaré dual of $\widetilde{\Sigma}_{a}$ and $\widetilde{x}$.

In the previous equations we have used the following definitions $x=-2 c_{1}(L \otimes L) \in$ $H^{2}(M, \mathbb{Z})$ and $\widetilde{x}_{\mathbf{X}}=\int_{M} i_{\mathbf{X}}(x) \mu$ with $\mathbf{X}$ being the 2-vector field wrapping $\widetilde{x}$. 


\section{A Physical Interpretation}

In this paper we have assumed the existence of "diffused" cycles in a suitable fourmanifold. Due their relevance in dynamical systems on simply-connected 4-manifolds we have considered asymptotic 2-cycles $\widetilde{\Sigma}_{\mathbf{Y}_{1}}, \ldots, \widetilde{\Sigma}_{\mathbf{Y}_{r}}$ together with a set of invariant probability measures $\mu=\mu_{1}+\cdots+\mu_{r}$, with $\mu_{i}$ supported in $\widetilde{\Sigma}_{\mathbf{Y}_{i}}$ on $M$. The present section is devoted to interpret this system in terms of string theory. Thus one would wonder if these 2-cycles can be interpreted as closed string probes propagating on the underlying four-manifold (which would be compact or non-compact). For this issue there is a nice response through the computation of the scattering amplitudes of an axion at zero momentum with a NS5-brane in the heterotic string theory [24]. In the present paper we follow this direction and we argue that Eq. (36) is a consequence of these considerations.

Let us consider a spacetime manifold $M^{1,9}$ provided with a set of Borel probability measures invariant under a non-singular smooth 2-flow generated by a 2 -vector field $Y$. Moreover we take the following splitting $M^{1,9}=M^{1,5} \times M$, where $M^{1,5}$ is a flat Minkowski space and $M$ is the transverse space. We consider a NS fivebrane (NS5) as a solitonic object filling the space $M^{1,5}$. Thus the transverse space $M$ consists of a four-manifold parameterizing the positions of the NS5-brane. Our flow 2-orbit can be regarded as a closed string propagating on $M^{1,9}$ without necessarily being localized in a homology cycle of $M^{1,9}$. For the purposes of this paper we focus on a special situation by limiting the 2-flow to be defined only in the transverse space $M$ and supported in the whole $M$. Consequently the Borel measures will be defined only on $M$. In this case we can think of the 2-flow as an asymptotic cycle $\widetilde{\Sigma}_{\mathbf{Y}}$ ) representing a "diffuse" closed string propagating in $M$ and viewing the NS5 as a scattering center. If the probability measures are totally on $M$ then the NS5-brane sector will remain unchanged and the moduli space of instantons remain the same. The effective action described in [24] is a non-linear sigma model on the worldvolume of the NS5 brane $W$ and with target space the space $\mathcal{M}_{N}$. This latter space represents the space of static NS5 brane solutions and it is equal to the moduli space of $N$ Yang-Mills instantons $\mathcal{M}_{N}(M)$ over $M$. Thus the ground states correspond to cohomology classes on $\mathcal{M}_{N}(M)$. If we identify the "diffuse" heterotic closed string with the asymptotic 2-cycles on $M$ i.e. $\widetilde{\Sigma}_{\mathbf{Y}}$, then the action is given by:

$$
S=\int_{M} i_{\mathbf{Y}}(B) \mu
$$

Then from the interacting terms coupling the $B$-field with the gauginos of the heterotic supergravity action we have

$$
\widetilde{O}_{i j, \mathbf{Y}}=\int_{M} i_{\mathbf{Y}}\left(Z_{i j}\right) \mu
$$

where $Z=\operatorname{tr}\left(\delta_{i} A \wedge \delta_{j} A-\phi_{i j} F\right)$.

Now we would like to consider multiple axion scattering with zero momentum. Then the transitions among the quantum ground states of the worldvolume $W$, for instance, from $|0\rangle$ to $|m\rangle$, induced by the scattering of $r$ axions with the NS5-branes is described by the scattering amplitude. The $r$ axions represent $r$ closed heterotic 
string wrapping the homology cycles $\widetilde{\Sigma}_{\mathbf{Y}_{1}}, \ldots, \widetilde{\Sigma}_{\mathbf{Y}_{r}}$, associated with the 2-vector fields $\mathbf{Y}_{1}, \ldots, \mathbf{Y}_{r}$. Thus the scattering amplitude is given by

$$
\begin{gathered}
\mathcal{A}\left(\mathbf{Y}_{1}, \cdots, \mathbf{Y}_{r}\right)=\left\langle m\left|\widetilde{O}_{\mathbf{Y}_{1}} \cdots \widetilde{O}_{\mathbf{Y}_{r}}\right| 0\right\rangle \\
=\int_{\mathcal{M}_{N}} \widetilde{O}_{\mathbf{Y}_{1}} \wedge \cdots \wedge \widetilde{O}_{\mathbf{Y}_{r}} .
\end{gathered}
$$

Thus we have deduced eq. (36) from string theory.

Above, we assumed that the set of Borel measures are distributed along the space $M$. This is consistent with the fact we have in the transverse directions (along $M^{1,5}$ ) filled with the NS5-brane which is a solitonic object and consequently is very heavy in the perturbative regime and consequently they are very difficult to excite.

S-duality between the heterotic and type I string interchanges NS5 by a D5-brane and axions by D1-branes [46]. The self-dual gauge field on $M$ leads to the ADHM construction of instantons [47]. The interactions are now given by the Type I string action. It would be interesting to make a description of the asymptotic cycles within this context.

\section{$9 \quad$ Final Remarks}

In the present paper we look for the implementation of the procedure followed in Ref. [22] for Jones-Witten invariants, to compute invariants for flows in higher dimensional manifolds. In the this situation the relevant invariants of interest were the smooth invariants of four-manifolds i.e. the Donaldson-Witten and the Seiberg-Witten invariants. We were able to obtain these invariants when some flows generated by non-singular and non-divergence-free smooth $p$-vector fields are globally defined on the four-manifold. We assumed that the homology cycles of $M$ are described by the asymptotic cycles. We focus our work on simply-connected four-manifolds, thus the only relevant flows are the 2-flows, though the invariants can be defined for any other $p$ flows. This is the situation that leads to a generalization of invariants of four-manifolds with flows.

In order to implement the above considerations we use the Witten cohomological field theory, whose observables are cohomology classes of $M$. In the presence of flows these observables were constructed as geometric currents underlying asymptotic cycles and foliations introduced by Ruelle and Sullivan [19] and Schwartzman [18]. Thus the asymptotic observables and their correlation functions give rise to new smooth invariants for four-manifolds with a dynamical system with an invariant probability measure. That is, they represent smooth invariants for foliations i.e. triples $(M, \mathcal{F}, \mu)$. This was done for Donaldson-Witten invariants (36) as well as for Seiberg-Witten invariants (79). Donaldson-Witten invariants are also obtained for the case of Kähler manifolds with flows and some examples were described in Sec. 5.3. Finally, we attempt

to give a physical interpretation in terms of string theory. We used the procedure outlined in Ref. [24] to obtain the invariants (36) as scattering amplitudes of $r$ axions at the zero momentum with $N$ coincident NS5-branes in the heterotic string theory. 
These axions are of special character and they represent $r$ 2-flows wrapping $r$ homology 2-cycles of $M$. We gave only general remarks on this subject and a further detailed analysis must be performed. This includes the incorporation of S-duality between the heterotic and Type I string theory [46] and the uses of this structure [47] to construct an ADHM construction of instantons with flows. It would be interesting to include flows in terms of proper dynamical fields such as is the case in string theory such as the NS $B$-field and the RR fields and make similar consideration as the present paper, but this time, in terms of specific interactions of the flow degrees of freedom. In this case it is possible to compute the back reaction of the fields of the theory to the flow.

There is a number of possible further generalizations of our work. One of them is the extension of asymptotic invariants to quantum cohomology [13, 14, 48], by considering asymptotic cycles in the target space of a topological non-linear sigma model of types $A$ or $B$ [14]. Of special interest is the possibility to define an asymptotic version of the Rozansky-Witten invariants [49]. This is due to the fact that their construction involves a topological sigma model on a 3-manifold and target space being an hyperKähler manifold. The theory leads to link-invariants on the 3-manifold with underlying structure group labeled by the hyper-Kähler structure. We would like to establish a relation with the results obtained in Ref. [22].

Another possibility is the consideration of flows generated by $p$-vector fields on supermanifolds. The analysis involves the computation of correlation functions with even and odd operators. This will constitute a supersymmetric extension of the work considered in the present paper. One more possible direction constitutes the implementation of the procedure to the computation of correlation functions of observables in the eight-dimensional generalization of the cohomological field theory [50, 51]. Some of these issues are already under current investigation and will be reported elsewhere.

\section{Acknowledgments}

We would like to thank the referee for carefully reading our manuscript and for giving us very important suggestions. The work of H. G.-C. is supported in part by the CONACyT grant 128761 . The work of R.S. was supported by a CONACyT graduate fellowship. The work of A.V. was partially supported by CONACyT grant number 129280 and DGAPA-PAPIIT, Universidad Nacional Autónoma de México.

\section{References}

[1] E. Witten, 'Quantum field theory and the Jones polynomial", Commun. Math. Phys. 121, 351 (1989).

[2] E. Witten, "Fivebranes and knots," arXiv:1101.3216 [hep-th]. 
[3] S.K. Donaldson, "Polynomial invariants for smooth 4-manifolds," Topology 29 (1990) 257.

[4] S.K. Donaldson and P.B. Kronheimer, The Geometry of four-manifolds, Oxford University Press, New York 1990.

[5] E. Witten, "Topological quantum field theory," Commun. Math. Phys. 117, 353386 (1988).

[6] E. Witten, "Topological gravity," Phys. Lett. B 206, 601 (1988); J. M. F. Labastida, M. Pernici and E. Witten, "Topological gravity in two-dimensions," Nucl. Phys. B 310, 611 (1988); E. Witten, "On the structure of the topological phase of two-dimensional gravity," Nucl. Phys. B 340, 281 (1990).

[7] K.G. O'Grady, "Donaldson polynomials for K3 surfaces", J. Diff. Geom. 35 (1992) 415-427.

[8] P.B. Kronheimer and T.S. Mrowka, "Recurrence relations and asymptotics for four-manifold invariants", Bull. Am. Math. Soc. 30 (1994) 215; "Embedded surfaces and the structure of Donaldson polynomial invariants", J. Diff. Geom. 41 (1995) 573 .

[9] E. Witten, "Supersymmetric Yang-Mills theory on a four-manifold", J. Math. Phys. 35 (10) 5101-5135 (1994).

[10] N. Seiberg and E. Witten, "Electric-magnetic duality, monopole condesation, and confinement in $\mathcal{N}=2$ supersymmetric Yang-Mills theory", Nucl. Phys. B 426, 19 (1994); erratum, ibid. B 430, 485 (1994); "Monopoles, duality and chiral symmetry breaking in $\mathcal{N}=2$ supersymmetric QCD", Nucl. Phys. B 431, 484 (1994).

[11] E. Witten, "Monopoles and four manifolds", Math. Res. Lett. 1769 (1994).

[12] J.M. Labastida and M. Mariño, Topological quantum field theory and four manifolds, Springer Verlag (2005).

[13] E. Witten, "Topological sigma models," Commun. Math. Phys. 118, 411 (1988).

[14] E. Witten, "Mirror manifolds and topological field theory," arXiv:hep-th/9112056.

[15] M. Mariño, Chern-Simons theory, matrix models, and topological strings, Oxford, UK: Clarendon (2005) 197 p.

[16] V.I. Arnold and B.A. Khesin, Topological methods in hydrodynamics, Springer Verlag (1998).

[17] S. Schwartzman, "Asymptotic cycles", Ann. Math. 66(2), 270-284 (1957).

[18] S. Schwartzman, "Higher dimensional asymptotic cycles," Canad. J. Math. 55(3), 636-648 (2003). 
[19] D. Ruelle and D. Sullivan, "Currents, flows and diffeomorphisms," Topology 14 319-327 (1975).

[20] D. Sullivan, "Cycles for the dynamical study of foliated manifolds and complex manifolds," Invent. Math. 36 225-255 (1976).

[21] S. Schwartzman, "Asymptotic cycles on non-compact spaces", Bull. London Math. Soc. 29, 350-352 (1997).

[22] A. Verjovsky, R. Vila Freyer, "The Jones-Witten invariant for flows on a 3Dimensional manifold", Commun. Math. Phys. 163 73-88 (1994).

[23] H. García-Compeán and R. Santos-Silva, "Link invariants for flows in higher dimensions," J. Math. Phys. 51, 063506 (2010).

[24] J. A. Harvey and A. Strominger, "String theory and the Donaldson polynomial," Commun. Math. Phys. 151, 221 (1993).

[25] G. de Rham, Differentiable manifolds: forms, currents, harmonic Forms, SpringerVerlag Berlin Heidelberg 1984.

[26] A. Candel, L. Conlon, Foliations I, Graduate Studies in Mathematics, 23. American Mathematical Society, Providence, RI, 2000.

[27] J. Plante, Foliations with measure preserving holonomy, Annals of Math. Second Series, Vol. 102, No. 2 (Sep., 1975), pp. 327-361.

[28] M.F. Atiyah, N.J. Hitchin and I.M. Singer, "Self-duality in four-dimensional Riemannian geometry", Proc. R. Soc. Lond. A 362 (1978) 425.

[29] H. Kanno, "Weyl algebra structure and geometrical meaning of BRST transformation in topological quantum field theory," Z. Phys. C 43, 477 (1989).

[30] M. F. Atiyah, L. Jeffrey, "Topological Lagrangians and cohomology", J. Geom. Phys. 7 (1) (1990) 120.

[31] V. Mathai and D. Quillen, "Superconnectons, Thom classes and equivariant differential forms", Topology 25 (1986) 85.

[32] I. Vaisman, Lectures on the geometry of Poisson manifolds, (Boston, MA: Birkhauser, 1994).

[33] M. Holm, "New insights in brane and Kaluza-Klein theory through almost product structures", arXiv:hep-th/9812168v1.

[34] J. S. Park, "N $=2$ topological Yang-Mills theory on compact Kahler surfaces," Commun. Math. Phys. 163, 113 (1994).

[35] P. C. Argyres and A. E. Faraggi, "The vacuum structure and spectrum of $\mathcal{N}=2$ supersymmetric $S U(n)$ gauge theory," Phys. Rev. Lett. 74, 3931 (1995). 
[36] G. van der Geer, Hilbert modular surfaces. Ergebnisse der Mathematik und ihrer Grenzgebiete. 3, 16. Springer-Verlag, Berlin, 1988.

[37] A. Selberg, On discontinuous groups in higher-dimensional symmetric spaces. Contributions to function theory (internat. Colloq. Function Theory, Bombay, 1960) pp. 147-164. Tata Institute of Fundamental Research, Bombay.

[38] F. Hirzebruch, "Hilbert modular surfaces", Enseignement Math. (19) No.2 (1973), pag. 183-281.

[39] R. Friedman, J. Morgan, Smooth four-manifolds and complex surfaces, Ergebnisse der Mathematik und ihrer Grenzgebiete (3), 27. Springer-Verlag, Berlin, 1994.

[40] S. K. Donaldson, "Lefschetz pencils on symplectic manifolds," J. Diff. Geom. 53 (1999), no. 2, 205-236.

[41] H.B. Lawson and M.-Louise Michelsohn, Spin geometry, (Princeton University Press, Princeton, 1989), Math. Series No. 39.

[42] D. Salamon, Spin geometry and Seiberg-Witten invariants, University of Warwick (1995).

[43] J. M. F. Labastida and M. Marino, "A topological Lagrangian for monopoles on four manifolds," Phys. Lett. B 351, 146 (1995).

[44] J. M. F. Labastida and M. Marino, "Non-abelian monopoles on four manifolds," Nucl. Phys. B 448, 373 (1995).

[45] J. M. F. Labastida and M. Marino, "Polynomial invariants for $S U(2)$ monopoles," Nucl. Phys. B 456, 633 (1995).

[46] J. Polchinski and E. Witten, "Evidence for heterotic - type I string duality," Nucl. Phys. B 460, 525 (1996).

[47] E. Witten, "Small instantons in string theory," Nucl. Phys. B 460, 541 (1996).

[48] M. Kontsevich and Yu. Manin, "Gromov-Witten classes, quantum cohomology, and enumerative geometry," Commun. Math. Phys. 164, 525 (1994).

[49] L. Rozansky and E. Witten, "Hyper-Kähler geometry and invariants of threemanifolds," Selecta Math. 3, 401 (1997).

[50] L. Baulieu, H. Kanno and I. M. Singer, "Special quantum field theories in eight and other dimensions," Commun. Math. Phys. 194, 149 (1998).

[51] B. S. Acharya, J. M. Figueroa-O'Farrill, B. J. Spence and M. O'Loughlin, "Euclidean D-branes and higher-dimensional gauge theory," Nucl. Phys. B 514, 583 (1998). 